\title{
Aspects of vineyard vegetation in North- eastern Italy and eastern neighbouring territories: Cerastio tenoreani-Geranietum dissecti and Mercurialetum annuae as archaic, disappearing coenosis
}

\author{
Livio Poldini ${ }^{1} \&$ Stefano Tasinazzo ${ }^{2 *}$ (1)
}

Key words: Cerastio tenoreaniGeranietum dissecti, Friuli Venezia Giulia, Italy, Mercurialetum annuae, phytosociology, Stellarietea mediae, Slovenia, south-eastern Europe, synsystematics, Veneto.

Ključne besede: Cerastio tenoreaniGeranietum dissecti, Furlanija-Julijska krajina, Italija, Mercurialetum annuae, fitosociologija, Stellarietea mediae, Slovenija, jugovzhodna Evropa, sinsistematika, Benečija.

Received: 3. 9. 2018

Revision received: 18.11 .2018

Accepted: 20. 11. 2018

\begin{abstract}
Recent original data concerning vineyard spring vegetation from Veneto hilly belt (North-eastern Italy) highlighted once again the phytogeographic originality of south-eastern territories with respect to Central European ones, also in anthropogenic coenosis. New relevés suggested to restate the association Cerastio tenoreani-Geranietum dissecti as vicarious vegetation of Geranio rotundifoliiAllietum vinealis occurring on the north side of the Alps. The comparison of autumn material from terracing vineyards of the sandy-marly Eocene flysch around Trieste coast with similar European relevés enabled to reject the not validly described Anagallido-Mercurialetum and to replace it with Mercurialetum annuae. In modern winegrowing Cerastio-Geranietum and Mercurialetum annuae are vanishing due to changing of agronomic schemes, as some relevés reported in the text document.
\end{abstract}

\begin{abstract}
Izvleček
$\mathrm{Na}$ osnovi novejših originalnih podatkov o spomladanski vegetaciji vinogradov $\mathrm{v}$ gričevnatem predelu Benečije (severovzhodna Italija) lahko ponovno izpostavimo fitogeografsko posebnost jugovzhodnih območij v primerjavi s Srednjo Evropo tudi, ko govorimo o antropogenih združbah. $\mathrm{Z}$ novimi popisi smo ponovno lahko ovrednotili asociacijo Cerastio tenoreani-Geranietum dissecti kot vikarianto asociacije Geranio rotundifolii-Allietum vinealis, ki se pojavlja severno od Alp. S primerjavo jesenskih vegetacijskih popisov, narejenih na terasastih vinogradih na eocenskem flišu v okolici tržaške obale, s podobnimi popisi iz Evrope, smo lahko nadomestili nepravilno opisano asociacijo Anagallido-Mercurialetum z asociacijo Mercurialetum annuae. Zaradi modernega vinogradništva in spremenjene kmetijske politike sestoji asociacij Cerastio-Geranietum in Mercurialetum annuae izginjajo, kar je razvidno iz popisnega gradiva.
\end{abstract}




\section{Introduction}

In Europe vineyard vegetation developed over centuries under the influence of hoeing and ploughing that favoured the growth of well established archaic coenosis such as the classical Geranio-Allietum, gravitating in south-western Germany (Fischer 1983). Vineyard communities on base rich soils of Central and Western Europe are traditionally attribute to Veronico-Euphorbion within Stellarietea mediae. Nowadays traditional cultivation regimes gave way to minimum soil tilling techniques including superficial harrowing, mulching and herbicide applications, each one promoting different changes in basal species composition. Moderate variations in traditional agrotechnical management lead to small weed transformations leaving unchanged the Stellarietea-species dominance (defined 'agroforms'), whereas strong changes, such as mulching, support the substitution with grassland communities (Wilmanns 1989). Contrary to other central European Countries, in Italy there are no historical vineyard vegetation studies that allow comparisons on changes induced by the different agricultural framework. It's common knowledge that strips between the vine plant rows were planted with cereals or legumes (Hruby, 1935), a habit that has been able to promote the spread of the Stellarietea species. Italian vineyard vegetation has been little studied even in recent times, in particular in the temperate bioclimate where the only researches were conducted by Poldini (1980, 1989), Poldini et al. (1998), Baldoni et al. (2001), Andreucci et al. (2003) and Tasinazzo (2015).

The spring vegetation of vineyards from Italian and Slovenian Karst to Collio-Goriška Brda was initially classified by Poldini $(1980,1989)$ to Cerastio tenoreani-Geranietum dissecti, that was interpreted as vicarious south-eastern coenosis of central European Geranio rotundifolii-Allietum vinealis. Later the same author (Poldini et al. 1996) questioned his own conclusions on the basis of Slovenian researchers' contributes which attributed Vipavska valley (Seljak 1989) and Koper (Kaligarič 1992) vineyard vegetation to Geranio-Allietum, but at the same time remarking the strong floristic and biogeographic differences between south-eastern and central european provenances. Finally, Poldini et al. (1998) concluded that Friuli Venezia Giulia vineyards could be included in a geographical race of Geranio-Allietum characterised by several termophilous species such as Alopecurus myosuroides, Calepina irregularis, Cerastium glomeratum, Cerastium brachypetalum/tenoreanum, Crepis vesicarialtaraxacifolia, Geranium dissectum, Lolium multiflorum, Poa sylvicola, Rumex crispus and Vicia sativa agg. Recent, original data gathered on Veneto hill reliefs and here analytically presented, suggest once again the distinctiveness of south-eastern vineyard spring veg- etation with respect to central European one, bringing again into question the framework of the south-eastern pre-Alpine vineyard vegetation (Tasinazzo 2015).

Another archaic coenosis potentially developing in early autumn in vineyards is Mercurialetum annuae. In the 80s Poldini (1980) described, but not validly published, as Anagallido-Mercurialetum some relevés from the Trieste coast, mainly performed on terracings of the sandy-marly Eocene flysch with Seslerio-Quercetum pubescentis rubietosum peregrinae as potential vegetation. The framework of these relevés are here discussed.

\section{Materials and Methods}

As regards the vineyard spring vegetation, original data were collected according to Braun-Blanquet methodology (1964) during 2010-2016 springtime (april-may) from hilly reliefs of Veneto, except one from eastern Lombardy (North-eastern Italy; rel. 43 in Table 2). The modified quantitative scale 2a, 2b of Barkman et al. (1964) was implemented for the assessment of species covers. Relevés were preferentially performed inside predominantly traditional vineyards with typical weeds occurring despite current soil cultivation techniques. Part of investigated plots were also checked in late summer-early autumn to follow temporal changes in floristic composition.

Sixty-three relevés were hierarchically classified by means of agglomerative clustering using Jaccard index applied to presence-absence data and complete-linkage method. Presence-absence data better reflect uncertainty and variability of floristic composition due to soil treatment, weed control method and tilling technique that change among years and stands. Following this elaboration, a 8-relevé group resulted well separated in the dendrogram. These 8 relevés were identified as referring to communities of non-calcareous substrates (conglomerate) or dynamically not related to Buglossoido purpurocaeruleae-Ostryion carpinifoliae sensu Poldini et al. (2017); given their distinctiveness they were removed from the dataset, reducing the relevés to 55 .

After removing once occurring species, a classification of the 55 stands from Veneto with available Geranio-Allietum s.l. analytical tables, by means of UPGMA method and Jaccard index applied to binary data, allowed recognizing homogeneity of original and literature data. At the same time, it was possible to detect disaggregating single outlier-relevés that were removed: two from Veneto and rel. 32-33-35 from Hungarian material, rel. 42 in Wilmanns \& Bogenrieder's work (1992) and rel. 3 from Seljak's table (1989). Data arranged according to the previous cluster analysis were used to obtain synthetic 
tables and consequently to compare different provenances of spring agroecophase vineyard vegetation. Further synthetic tables were also obtained from Oberdorfer (1993) and Orgis (col. 2b in Tab. 3, in Fischer (1983)), whereas other synthetic data from Germany were excluded because already computed in Oberdorfer's table and so was for Slovenian data by Šilc \& Čarni (2007) realistically including Kaligaričs stands (1992). As Orgis' material (Fischer 1983) was given in frequency classes it was inserted in Table 1, but it was not in Figure 2.

As cultivation techniques in viticulture has strong influences on composition of vineyard coenoses (Wilmanns 1989, Poldini et al. 1998), were selected for the analysis only relevés of Geranio-Allietum which belong to the 'typical form' according to authors' assertion and excluding agroforms sensu Poldini et al. (1998) and mixed stands ("Durchdringungen") or replacing communities due to mulching practice sensu Wilmanns (1989) and Wilmanns \& Bogenrieder (1991). Summer-developed species, for the most part C4, such as Amaranthus sp. pl., Chamaesyce sp. pl., Setaria sp. pl., Digitaria sanguinalis, Portulaca oleracea, Galinsoga sp. pl., Calystegia sepium ecc., were removed from comparison tables before undergo statistical analysis to unbias results. Their occurring, representing the very first symptoms of transition towards the late-summer agroecophase, was reported only by some of the cited papers, according to survey times.

Thus, from literature were selected the following data:

- Fischer (1983): column 2b in Tab. 3;

- Fischer (1983): Tab. 2;

- Wilmanns (1989): Tab. 1 rel. 1-16 representing the classic association of vineyards in Kaiserstuhl (D), whereas Poa trivialis-Lolio-Potentillion community stands and 'penetration forms' (sensu Author) were excluded;

- Seljak (1989): Tab. 4;

- Wilmanns \& Bogenrieder (1991): Tab. 19.2 rel. 1-7 representing 'old' Geranio-Allietum in Upper Rhine Lowland (D); mixed stands of Geranio-Allietum with Agrostietea species renamed penetrations (= Durchdringungen) by Authors were excluded from analysis;

- Wilmanns \& Bogenrieder (1992): Tab. 5 rel. 1-26 and 35-44, excluding impoverished ones (rel. 27-34);

- Kaligarič (1992): Tab. 2;

- Oberdorfer (1993): Tab. 149 column 25;

- Poldini et al. (1998): Tab. 4 rel. 1-35 representing the 'typical form' of Geranio-Allietum, excluding relevés attributed to Taraxacum officinale, Arrhenatherum elatius and Agrostis stolonifera agroforms;

- Pál (2006): Tab. 1.

As regards early autumn coenosis (Mercurialetum annuae), partly original and published relevés gathered in Italian Karst (Poldini 1980, 1989) were compared with
Mercurialetum annuae stands from Holland (Kruseman \& Vlieger 1939), Marche (Baldoni et al. 2001) and with big and more recent data from Germany (Oberdorfer 1993), Slovenia (Šilc \& Čarni 2007) and Czech Republic (Chytrý 2009) that outlined the diagnostic species set of the association.

Statistical analysis was conducted with Syn-Tax 2000 package (Podani 2001). For syntaxonomical scheme, we refer to the vegetation prodrome of Italy (Biondi et al. 2014).

Syntaxonomical classification of vegetation data were interpreted in agreement with the International Code of Phytosociological Nomenclature (Weber et al. 2000).

The floristic nomenclature follows Bartolucci et al. (2018) and Galasso et al. (2018), Euro+Med PlantBase Med-list (http://www.emplantbase.org) for species that are missing in Italy.

\section{Results and Disussion}

\section{Cerastio tenoreani-Geranietum dissecti}

The classification of original (Veneto) and available analytical data gathers together stands from Veneto (cluster Ala in Figure 1) and those from Friuli Venezia Giulia and Slovenia (Seljak 1989, Kaligarič 1992, Poldini et al. 1998, cluster A1b) at a higher similarity level than relevés from Germany (Fischer 1983, Wilmanns 1989, Wilmanns \& Bogenrieder 1991, 1992; cluster A2). Hungarian relevés separate at an even higher level (Pál 2006; cluster B) and according to author himself, they constitute an impoverished form of Geranio-Allietum, but maybe they could be attributed to an independent syntaxon on the basis of the occurrence of Holosteum umbellatum, Viola arvensis, Vicia grandiflora and Androsace maxima.

Synthetic data (Figure 2) show a higher similarity between north-eastern Italian and Slovenian than between different German provenances.

Classification of selected synthetic tables resulted in synoptic Table 1 . With the addition of data from Veneto, most species above reported and mentioned by Poldini et al. (1998) confirm their role as differential species of south-eastern vineyard vegetation that it is here proposed anew as Cerastio tenoreani-Geranietum dissecti. Geranium dissectum, Cerastium glomeratum, Calepina irregularis, Poa sylvicola, Lolium multiflorum, Crepis vesicarialtaraxacifolia and Cerastium brachypetalum/tenoreanum are differential species of the coenosis, the last two with Rumex crispus, Alopecurus myosuroides, Trifolium molinerii and Anthemis arvensis also distinguishing the more eastern new subassociation typicum. Ornithogalum divergens can be added to the differential species of the association; in the Medi- 


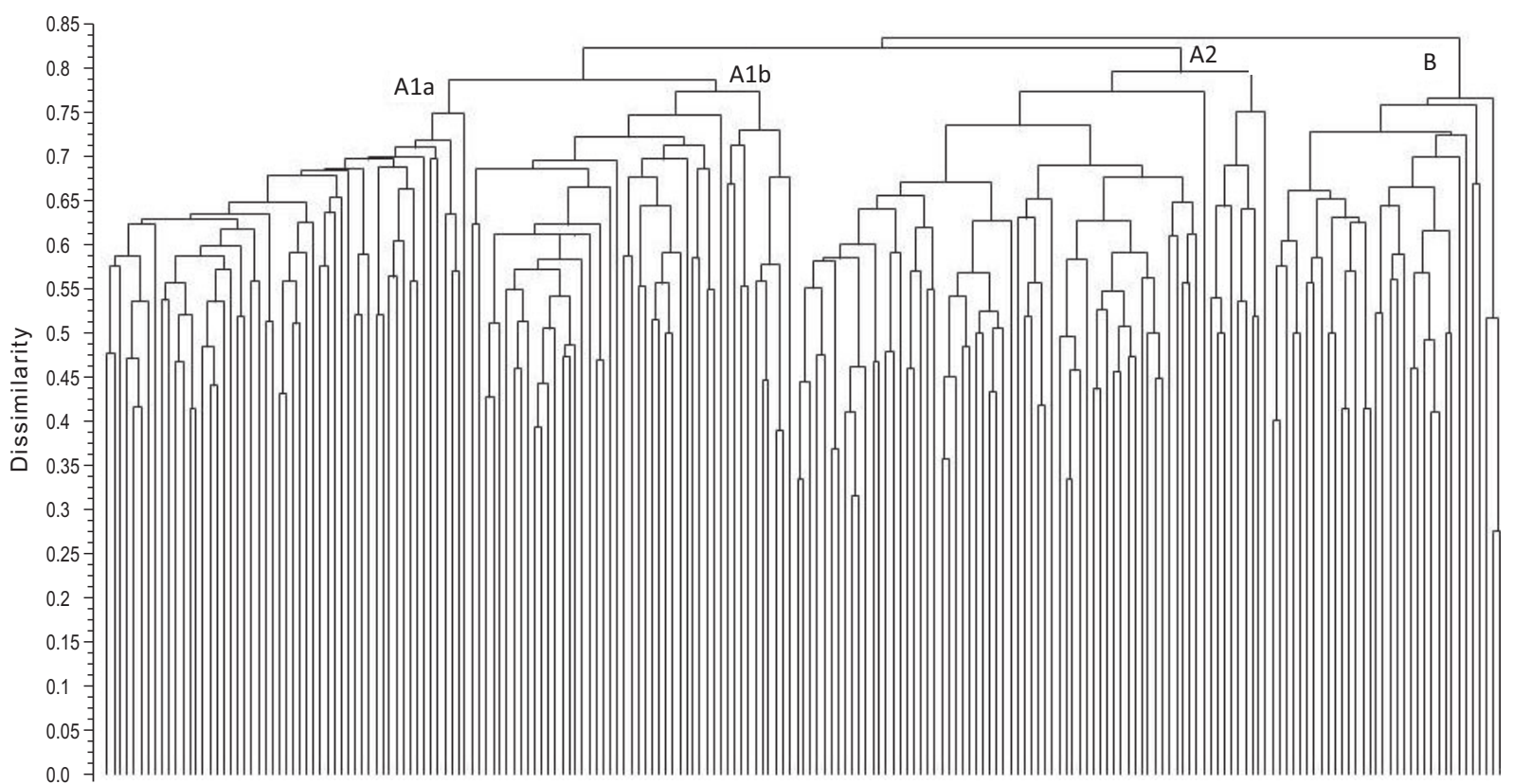

Figure 1: Classification of south-eastern and central European vineyard relevés (UPGMA-Jaccard without once occurring species, binary data). Cluster A1a: relevés from Veneto; cluster A1b: relevés from Friuli Venezia Giulia and Slovenia; cluster A2: relevés from Germany; cluster B: relevés from Hungary.

Slika 1: Klasifikacija jugovzhodnih in srednjeevropskih popisov vinogradov (UPGMA-Jaccard brez vrst, ki se pojavljajo samo enkrat, binarni podatki). Klaster A1a: popisi iz Benečije; klaster A1b: popisi iz Furlanije-Julijske krajine in Slovenije; klaster A2: popisi iz Nemčije; klaster B: popisi z Madžarske.

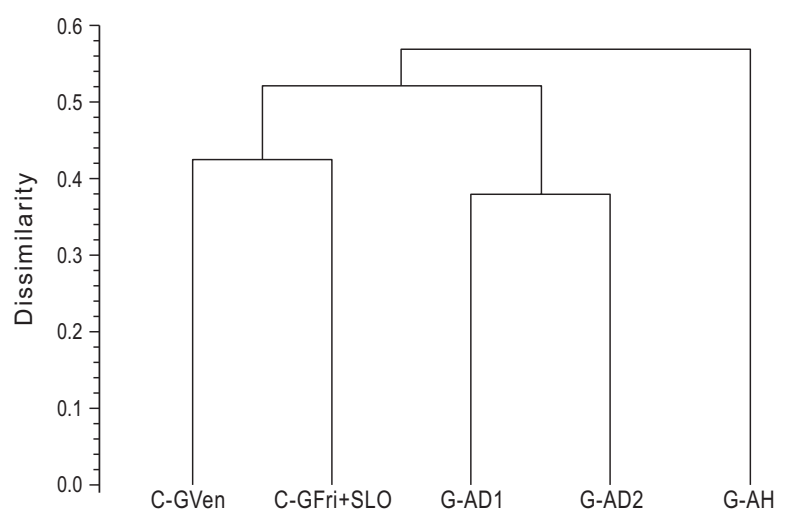

Figure 2: Classification of south-eastern and central European vineyard synthetic tables (UPGMA-similarity ratio without once occurring species). C-G: Cerastio-Geranietum; G-A: Geranio-Allietum; Ven: Veneto; Fri: Friuli Venezia Giulia; SLO: Slovenia; D1: cluster A2 in Figure 1; D2: Oberdorfer (1983); H: Hungary.

Slika 2: Klasifikacija jugovzhodnih in srednjeevropskih popisov vinogradov, urejenih v sintetski tabeli vinogradov (UPGMA-Jaccard brez vrst, ki se pojavljajo samo enkrat). C-G: Cerastio-Geranietum; G-A: Geranio-Allietum; Ven: Benečija; Fri: Furlanija-Julijska krajina; SLO: Slovenija; D1: klaster A2 v Sliki 1; D2: Oberdorfer (1983); $\mathrm{H}$ : Madžarska.

terranean context it replaces $O$. umbellatum thriving in central European vineyards. At the alliance level Veronica polita differentiates German and Hungarian relevés as it is totally absent from south-eastern stands.
From an ecological point of view, stands from Veneto have a distinctive feature in holding several high covering and very frequently occurring thermophilous species with a Mediterranean distribution, such as Crepis sanctal nemausensis and Geranium molle in V frequency class, Hordeum murinum/leporinum, Cynodon dactylon and Cardamine hirsuta in IV (Table 2). In particular, Crepis sanctalnemausensis, a recently and currently spreading species in Veneto, is usually so abundant to dominate the relevé (Figure 3). It was also indicated as differential species of Calendulo arvensis-Crepidetum sanctae a spring vineyard vegetation thriving in sub-Mediterranean variant of temperate bioclimate of Marche region in central Italy (Baldoni et al. 2001). Vineyard vegetation in Veneto is referred to the new subassociation crepidetosum nemausensis subass. nova hoc loco (holotypus: Table 2, rel. 3) occurring in hilly belt where it was registered from 25 to $370 \mathrm{~m}$ a.s.l. in territories influenced by a temperate-oceanic to temperate-continental bioclimate - often in the sub-Mediterranean variant - upper mesotemperate thermotype and prevalently upper subhumid ombrotype. Despite total lack of historical or also recent documentations on the past composition of Veneto vineyard vegetation, drastic changes in cultivation methods over last decades surely led to floristic substitutions as observed in Germany at least since the mid-80s (Wilmanns 1989, Wilmanns 


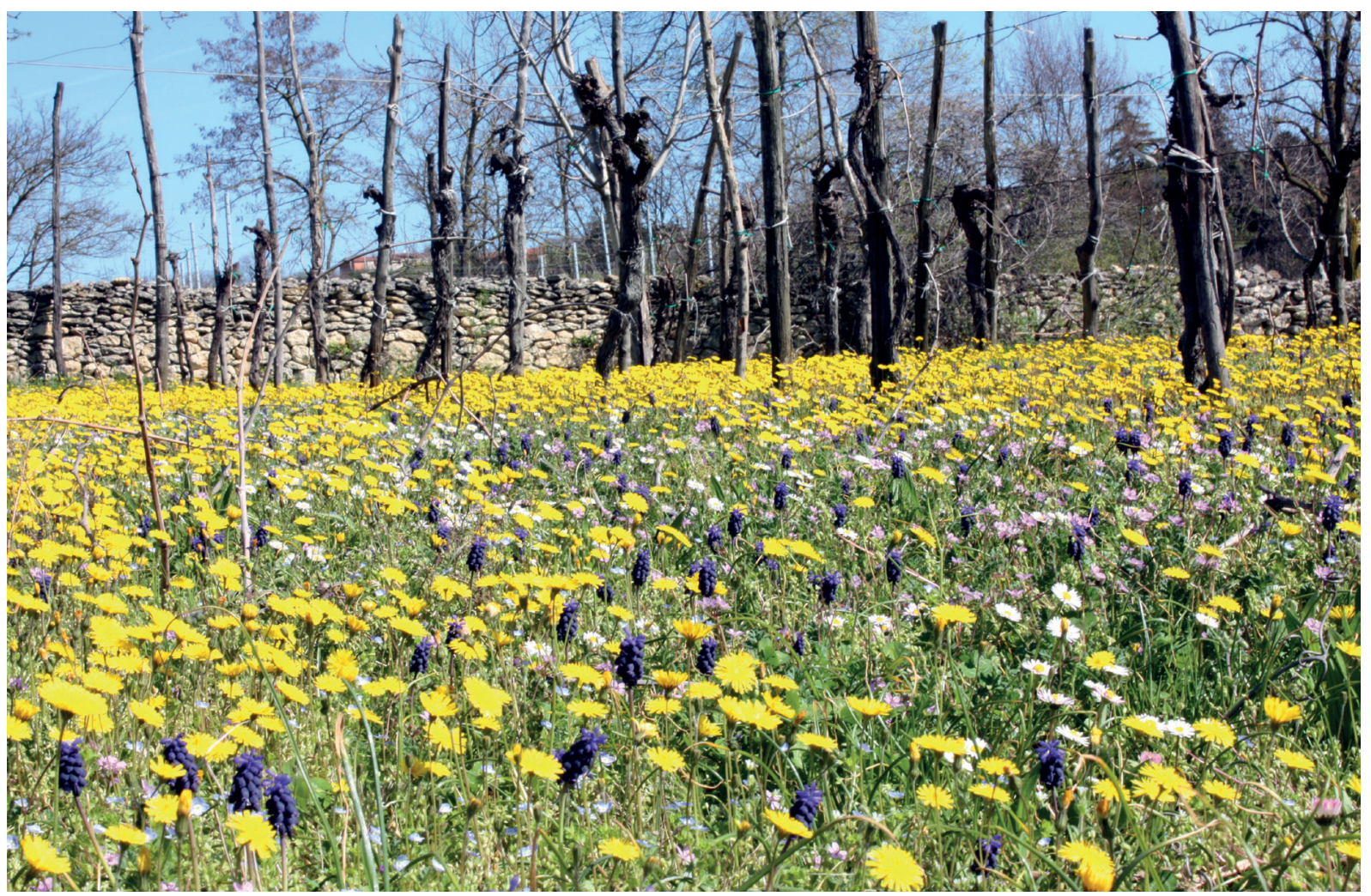

Figure 3: Stand of Cerastio-Geranietum subass. crepidetosum nemausensis (rel. 16, Colli Berici).

Slika 3: Sestoj subasociacije Cerastio-Geranietum subass. crepidetosum nemausensis (popis 16, Colli Berici).

\& Bogenrieder 1991). Our recent relevés dating back to 2010-2016 period show evident traces of the influence of minimum soil tilling techniques that nowadays replaced anachronistic cultivation with an active soil movement. Usually only few small plots for family use continue to be cultivated with rototillers, but superficial harrowing and mulching practices tend to affect most of stands by favouring the spreading of Cynosurion (Lolium perenne, Trifolium repens, Bellis perennis) or runner emitting species. Among these, Cynodon dactylon emphasizes warm environmental conditions though it only sprouts in spring as its vigorous growth is only from the summer. In addition, some rosulate hemicryptophytes manage to 'escape' from spring mechanical control (e.g. Rumex pulcher).

The association can be considered as vanishing because of not only changing in cultivation systems but also due to the diffusion of permanent green cover technique in newly planted or replanted vineyard by sowing cultivar of grasses like Lolium perenne and Lolium arundinaceum. Some relevés of this kind are shown in Table 3, where differences between provenances depend on used mixtures. This approach depresses Stellarietea elements that remain as sporadic relics especially under vine rows where spraying of herbicides contain them.
Cerastio-Geranietum is a spring association; in late summer an autumn agroecophase develops in the same vineyards when tilling are abandoned in the harvest proximity, most of Stellarietea therophytes have already disappeared and there is the spreading of $\mathrm{C} 4$ species. Covers or frequencies of some late-spring germinating species occurring in relevés (Table 2), such as Cynodon dactylon, Convolvulus arvensis, Polygonum aviculare agg., Chenopodium album etc., increase in late season, whereas summersprouting weeds enter as new the vineyard habitat. Data coming from September-October surveys in 40 out of the 53 stands performed in Veneto are given in Table 4 showing only strictly autumn weeds. This species set raises the occurring rate of Solano-Polygonetalia character species.

\section{Mercurialetum annuae}

Synthetic data analysis and the resulting simplified synoptic table are shown in Figure 4 and Table 5, respectively. Based on the occurrence of the diagnostic species Mercurialis annua, Solanum nigrum, Amaranthus retroflexus, Chenopodium album and Senecio vulgaris it is possible to refer the vegetation then collected from the Trieste coast and the Italian Karst to the Mercurialetum annuae. Among high frequency species of higher syntaxa there are 
Convolvulus arvensis, Bromus sterilis, Anagallis arvensis, Allium vineale, Capsella bursa-pastoris, Cirsium arvense, Fumaria officinalis.

Like Cerastio-Geranietum, in Italian Karst Mercurialetum annuae is almost everywhere disappeared and the abandoned terracings rapidly evolve towards an anthropogenic steppic-grassland (Brachypodio-Agropyretum intermedii) and then to a Rubus ulmifolius scrub (ClematidoRubetum).

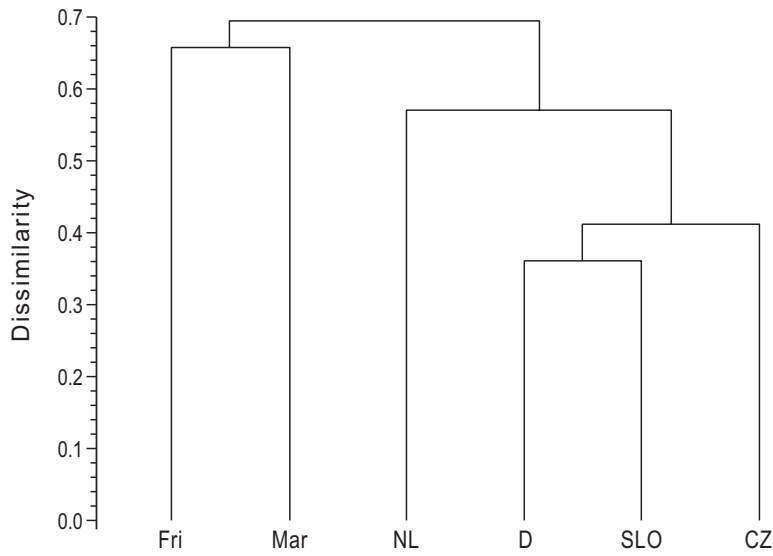

Figure 4: Classification (UPGMA-similarity ratio) of Mercurialetum annuae synthetic tables from southern and Central Europe. Fri: Friuli Venezia Giulia (North-eastern Italy); Mar: Marche (central Italy); NL: Nederland; D: Germany; SLO: Slovenia; CZ: Czech Rep. Slika 4: Klasifikacija (UPGMA-similarity ratio) sintetske tabele asociacije Mercurialetum annuae iz južne in Srednje Evrope. Fri: Furlanija-Julijska krajina (severovzhodna Italija); Mar: Marche (srednja Italija); NL: Nizozemska; D: Nemčija; SLO: Slovenija; CZ: Češka.

\section{Conclusions}

Springtime vegetation of south-eastern vineyards differ markedly from central European one by the high rate of species with Mediterranean and south-eastern distribution, whereas summer-autumn phenophases are not so much different. From the synphytosociological point of view, vineyards are the result of forest clearing belonging to very different series of vegetation in south-eastern and central Europe. As already stated by Poldini et al. (1998), in Friuli Venezia Giulia, Veneto and Slovenia xerotermophilous or xeromesophilous sub-Mediterranean Ostrya and Quercus-dominated woods are the mature stages of hilly vegetation. Despite simplifications induced by current agriculture, in south-eastern vineyard vegetation floristic peculiarities remain to highlight biogeographical differences that confirm the occurring of a vicarious coenosis of central European Geranio-Allietum, i.e. Cerastio-Geranietum. The latter as well as autumn Mercularietum annuae are nowadays disappearing coenosis due to modern wine growing that resort to mowing, non-tillage floor management and seeding of perennial grasses.

\section{Syntaxonomical scheme}

Stellarietea mediae Tüxen, Lohmeyer \& Preising ex von Rochow 1951.

\section{Stellarienea mediae}

Solano nigri-Polygonetalia convolvuli (Sissingh in Westhoff, Dijk, Passchier \& Sissingh 1946) O. Bolòs 1962.

Veronico agrestis-Euphorbion peplus Sissingh in Passarge 1964.

Cerastio tenoreani-Geranietum dissecti Poldini 1980 (Geranio-Allietum vinealis sensu Poldini et al. 1998 non Tüxen ex von Rochow 1951).

typicum subass. nova hoc loco

crepidetosum nemausensis subass. nova hoc loco.

Mercurialietum annuae Kruseman et Vlieger 1939 ex Westhoff et al. 1946. (corresponding name: Anagallido-Mercurialetum Poldini 1980, nom. inval. art. 5)

Other syntaxa quoted in the text:

Brachypodio-Agropyretum intermedii Poldini 1980

Buglossoido purpurocaeruleae-Ostryion carpinifoliae Poldini, Sburlino \& Vidali 2017

Calendulo arvensis-Crepidetum sanctae Baldoni, Biondi \& Loiotile 2001

Clematido-Rubetum Poldini 1980

Seslerio autumnalis-Quercetum pubescentis rubietosum peregrinae Zupančič 1999

\section{Acknowledgements}

We would like to thank dr. G. Seljak for giving his unpublished thesis data. We are grateful to two anonymous referees which improved the manuscript.

Stefano Tasinazzo (D), https://orcid.org/0000-0002-58290456 


\section{References}

Andreucci F., Bagliani C., Berta G. \& Castelli M. 2003: La vegetazione della Riserva Naturale Speciale della Val Sarmassa. Riv. Piem. St. Nat. 24: 3-65.

Baldoni M., Biondi E. \& Loiotile A. 2001: La vegetazione infestante i vigneti nelle Marche. Fitosociologia 38 (2): 63-68.

Barkman J.J., Doing H. \& Segal S. 1964: Kritische Bemerkungen und Vorschläge zur quantitativen Vegetationsanalyse. Acta Bot. Neerl. 13: 394-419.

Bartolucci F., Peruzzi L., Galasso G., Albano A., Alessandrini A., Ardenghi N. M. G., Astuti G., Bacchetta G., Ballelli S., Banfi E., Barberis G., Bernardo L., Bouvet D., Bovio M., Cecchi L., Di Pietro R., Domina G., Fascetti S., Fenu G., Festi F., Foggi B., Gallo L., Gottschlich G., Gubellini L., Iamonico D., Iberite M., JiménezMejías P., Lattanzi E., Marchetti D., Martinetto E., Masin R. R., Medagli P., Passalacqua N. G., Peccenini S., Pennesi R., Pierini B., Poldini L., Prosser F., Raimondo F. M., Roma-Marzio F., Rosati L., Santangelo A., Scoppola A., Scortegagna S., Selvaggi A., Selvi F., Soldano A., Stinca A., Wagensommer R. P., Wilhalm T., Conti F. 2018: An updated checklist of the vascular flora native to Italy. Plant Biosystems 152 (2): 179-303.

Biondi E., Blasi C., Allegrezza M., Anzellotti I., Azzella M. M., Carl E., Casavecchia S., Copiz R., Del Vico E., Facioni L., Galdenzi D., Gasparri R., Lasen C., Pesaresi S., Poldini L., Sburlino G., Taffetani F., Vagge I., Zitti S., Zivkovic L. 2014: Plant communities of Italy: the vegetation prodrome. Plant Biosystems 148 (4): 728-814.

Braun-Blanquet J. 1964: Pflanzensoziologie - Grundzüge der Vegetationskunde. Springer-Verlag, Wien, 865 pp.

Chytrý M. (ed.) 2009: Vegetace České republiky 2. Ruderální, plevelová, skalní a sutová vegetace / Vegetation of the Czech Republic 2. Ruderal, Weed, Rock and Scree vegetation. Academia, Praha, 520 pp.

Fischer A. 1983: Wildkrautvegetation der Weinberge des Rheingaus (Hessen): Gesellschaften, Abhängigkeit von modernen Bewirtschaftungs-methoden, Aufgaben des Naturschutzes. Phytocoenologia 11 (3): 331-383.

Galasso G., Conti F., Peruzzi L., Ardenghi N. M. G., Banfi E., Celesti-Grapow L., Albano A., Alessandrini A., Bacchetta G., Ballelli S., Bandini Mazzanti M., Barberis G., Bernardo L., Blasi C., Bouvet D., Bovio M., Cecchi L., Del Guacchio E., Domina G., Fascetti S., Gallo L., Gubellini L., Guiggi A., Iamonico D., Iberite M., JiménezMejías P., Lattanzi E., Marchetti D., Martinetto E., Masin R. R., Medagli P., Passalacqua N. G., Peccenini S., Pennesi R., Pierini B., Podda L., Poldini L., Prosser F., Raimondo F. M., Roma-Marzio F. Rosati L., Santangelo A., Scoppola A., Scortegagna S., Selvaggi A., Selvi F., Soldano A., Stinca A., Wagensommer R. P., Wilhalm T., Bartolucci F. 2018: An updated checklist of the vascular flora alien to Italy. Plant Biosystems 152 (3): 556-592.

Hruby J. 1935: La vegetazione delle colline a settentrione di Conegliano. Atti R. Ist. Ven. Sc. Lett. Arti 94: 461-483.

Kaligarič M. 1992: Vegetacija plevelov v vinogradih Koprskega primorja. Annales, ser. hist. natur, 2: 39-52.

Kruseman G. \& Vlieger J. 1939: Akkerassociaties in Nederland. Nederlandsch Kruidkundig Archiv 49: 327-98.
Oberdorfer E. 1993: Süddeutsche Pflanzengesellschaften. Teil III. Wirtschaftswiesen und Unkrautgesellschaften. 3. Auflage. G. Fischer Verlag, Jena.

Orgis K. 1977: Die Weinbergunkrautgesellschaften im Gebiet des mittleren Keupers in Franken besonders im Hinblick auf die Auswirkungen der Flurbereinigung. Hoppea 36: 193-246.

Pál R. 2006: Verbreitung und Assoziationsverhältnisse von Zwiebelgeophyten in den Weinbergen Süd-Ungarns. Journal of Plant Diseases and Protection 20: 619-626.

Podani J. 2001: Syn-Tax 2000. Computer program for data analysis in ecology and ystematics. User's manual. Scientia Publishing, Budapest.

Poldini L. 1980: Übersicht über die Vegetation des Karstes von Triest und Görz (NO-Italien). Studia Geobotanica 1(1): 79-130.

Poldini L. 1989: La vegetazione del Carso isontino e triestino. Lint, Trieste, $320 \mathrm{p}$.

Poldini L., Mazzolini G. \& Oriolo G. 1996: La vegetazione spontanea dei vigneti nei territori nord-est adriatici: Geraniorotundifolii-Allietum $\mathrm{R}$. Tx. ex von Rochow 1951 o CerastioGeranietum dissecti Poldini 1980? Giorn. Bot. Ital. 130(1): 151-152.

Poldini L., Oriolo G. \& Mazzolini G. 1998: The segetal vegetation of vineyards and crop fields in Friuli-Venezia Giulia (NE Italy). Studia Geobotanica 16: 5-32.

Poldini L., Sburlino G. \& Vidali M. 2017: New syntaxonomic contribution to the vegetation prodrome of Italy. Plant Biosystems 151(6): 1111-1119.

Seljak G. 1989: Plevelna vegetacija vinogradov in sadovnjakov na Goriškem in vpliv večletne rabe nekaterih herbicidov na spremembo dominantnosti plevelnih vrst. Biotehniška fakulteta v Ljubljani. (magistrsko delo). [in Slovenian].

Šilc U. \& Čarni A. 2007: Formalized classification of the weed vegetation of arable land in Slovenia. Preslia 79: 283-302.

Tasinazzo S. 2015: Sulla vegetazione dei vigneti collinari del Veneto. In $49^{\circ}$ Congresso S.I.S.V. "La scienza della vegetazione per la biodiversità e la sostenibilità”, Ancona 24-26 settembre 2015, abstract: 34

Weber H.E., Moravec J. \& Theurillat J.P. 2000: International Code of Phytosociological Nomenclature. 3rd edition. Journal of Vegetation Science 11: 739-768.

Wilmanns O. 1989: Vergesellschaftung und Strategie-Typen von Pflanzen mitteleuropäischer Rebkulturen. Phytocoenologia 18(1): 83-128.

Wilmanns O. \& Bogenrieder A. 1991: Phytosociology in vineyards results, problems, tasks. In Esser G. \& Overdieck D. (eds.), Modern ecology: basic and applied aspects: 399-441. Elsevier, AmsterdamLondon-New York-Tokyo.

Wilmanns O. \& Bogenrieder A. 1992. Das Geranio-Allietum in der oberelsässischen Rebflur. Bauhinia 10: 99-114. 
Appendix I: localities, dates, quadrants and geographical coordinates of relevés of Table 2 Cerastio-Geranietum crepidetosum nemausensis.

Rel. 1: C. Targon (Brendola-Colli Berici-VI), 29/03-

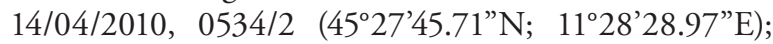
rel. 2: Terrossa (Roncà-M. Lessini-VR), 7/04-

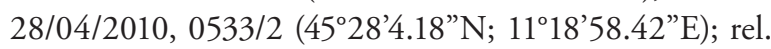
3: Gambugliano (M. Lessini-VI), 11/04/2016, 0434/2 $\left(45^{\circ} 35^{\prime} 31.02^{\prime \prime N}\right.$; $\left.11^{\circ} 25^{\prime} 48.15^{\prime \prime} \mathrm{E}\right)$; rel.4: Perarolo (Arcugnano-Colli Berici-VI), 30/03-13/04/2010, 0534/2 $\left(45^{\circ} 28^{\prime} 24.42^{\prime \prime} \mathrm{N}\right.$; $\left.11^{\circ} 29^{\prime} 46.52^{\prime \prime} \mathrm{E}\right)$; rel. 5: Turri (Montegrotto Terme-Colli Euganei-PD), 12/04-19/05/2011,

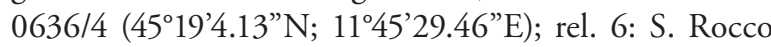
(Lavagno-M. Lessini-VR), 15/04-20/5/2011, 0433/2 $\left(45^{\circ} 26^{\prime} 57.19^{\prime \prime} \mathrm{N}\right.$; $11^{\circ}$ 6'54.54”E); rel. 7: S. Valentino (Brendola-Colli Berici-VI), 14/04-25/05/2010, 0534/2 $\left(45^{\circ} 28^{\prime} 12.55^{\prime \prime N}\right.$; $\left.11^{\circ} 28^{\prime} 50.36^{\prime \prime} \mathrm{E}\right)$; rel. 8: via Moschine (Baone-Colli Euganei-PD), 08/04-19/05/2011, 0736/1 (4515'13.72”N; 1141'46.69”E); rel. 9: Rocca di Lonigo (Colli Berici-VI), 31/03-10/05/2010, 0634/1 $45^{\circ} 23^{\prime} 39.97^{\prime \prime} \mathrm{N}$; $\left.11^{\circ} 24^{\prime} 23.41^{\prime \prime} \mathrm{E}\right)$; rel. 10: S. Briccio (Lavagno-M. Lessini-VR), 15/04-20/5/2011, 0532/4 $\left(45^{\circ} 26^{\prime} 46.25^{\prime \prime} \mathrm{N}\right.$; $\left.11^{\circ} 7^{\prime} 17.61^{\prime \prime} \mathrm{E}\right)$; rel. 11: Le Acque (Lonigo-Colli Berici-VI), 31/03-10/05/2010, 0634/1 $\left(45^{\circ} 23^{\prime} 27.84 ” N ; \quad 11^{\circ} 24^{\prime} 49.36^{\prime \prime} \mathrm{E}\right)$; rel. 12: Monticello (Lonigo-Colli Berici-VI), 31/03-10/05/2010,

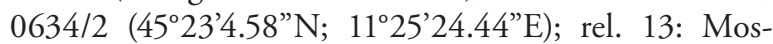
sano (Colli Berici-VI), 05/04-18/05/2010, 0535/3

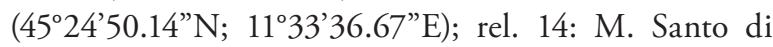
Lovertino (Albettone-Colli Euganei-VI), 16/04/2016,

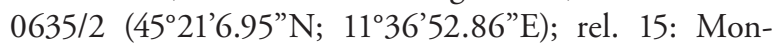
ticello (Lonigo-Colli Berici-VI), 31/03-10/05/2010, 0634/2 ( $45^{\circ} 23^{\prime} 5.22^{\prime \prime N}$; $\left.11^{\circ} 25^{\prime} 12.56^{\prime \prime} \mathrm{E}\right)$; rel. 16: Peraro (Lonigo-Colli Berici-VI), 06/04-12/05/2010, 0634/2

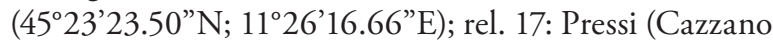
di Tramigna-M. Lessini-VR), 15/04-20/05/2011, 0533/1 $\left(45^{\circ} 27^{\prime} 16.93^{\prime \prime N}\right.$; $\left.11^{\circ} 13^{\prime} 3.39^{\prime \prime} \mathrm{E}\right)$; rel. 18: S. Ambrogio Valpolicella (M. Lessini-VR), 05/04-20/05/2011, 0431/3 (4531'44.28”N; 1050'48.61”E); rel. 19: Brendola (Colli Berici-VI), 14/04-25/05/2010, 0534/2 (45⒉'14.46”N; $11^{\circ} 27^{\prime} 1.52$ "E); rel. 20: Valcerea (Castelnuovo del Garda-morainic hills-VR), 30/03-25/05/2012, 0530/4 $\left(45^{\circ} 24^{\prime} 55.74 " \mathrm{~N} ; \quad 10^{\circ} 45^{\prime} 6.13^{\prime \prime} \mathrm{E}\right)$; rel. 21: Carbonara (Rovolon-Colli Euganei-PD), 19/04/2016, 0635/2 $\left(45^{\circ} 21^{\prime} 53.43\right.$ "N; $\left.11^{\circ} 38^{\prime} 35.19^{\prime \prime} \mathrm{E}\right)$; rel. 22: S. Daniele (Lonigo-Colli Berici-VI), 31/03-10/05/2010, 0634/1 $\left(45^{\circ} 23^{\prime} 2.55^{\prime \prime} \mathrm{N}\right.$; $\left.11^{\circ} 24^{\prime} 38.81^{\prime \prime E}\right)$; rel. 23: Ca Vecchie (Alonte-Colli Berici-VI), 06/04-10/05/2010, 0634/2 $\left(45^{\circ} 22^{\prime} 33.28^{\prime \prime} \mathrm{N} ; \quad 1^{\circ} 27^{\prime} 28.10^{\prime \prime} \mathrm{E}\right)$; rel. 24: cimitero di Selva (Montebello Vicentino-Lessini-VI), 07/04-

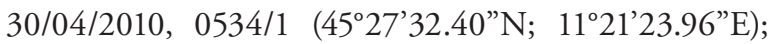

rel. 25: Recoaretto (Soave-M. Lessini-VR), 09/04/2010,

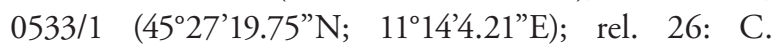
Ronca (Villaga-Colli Berici-VI), 06/04-14/05/2010,

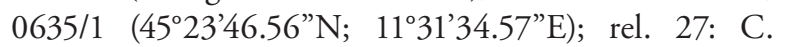
Agriman (Barbarano Vicentino-Colli Berici-VI),

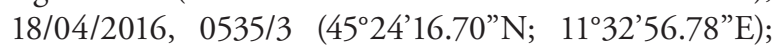
rel. 28: Noseo (Barbarano Vicentino-Colli Berici-VI), 18/04/2016, 0535/3 (4524'20.97”N; 1131'52.73”E); rel. 29 S. Lucia (M. Rusta-Colli Euganei-PD),

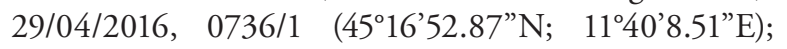
rel. 30: S. Silvestro (Boccon-Colli Euganei-PD),

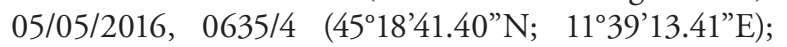
rel. 31: Marcellise (M. Lessini-VR), 06/05/2016, 0532/2 (45⒉'19.30”N; $11^{\circ}$ 5’46.68” E); rel. 32: Barbarano Vicentino (Colli Berici-VI), 05/04-18/05/2010,

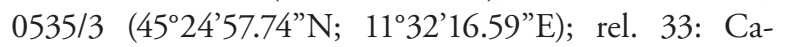
stelcerino (Soave-M. Lessini-VR), 09/04/2010, 0533/1 $\left(45^{\circ} 27^{\prime} 22.40^{\prime \prime} \mathrm{N}\right.$; 11 $\left.14^{\prime} 48.05^{\prime \prime} \mathrm{E}\right)$; rel. 34: Mozzarelli (Roncà-M. Lessini-VR), 09/04-21/05/2010, 0533/2 $\left(45^{\circ} 29^{\prime} 44.30^{\prime \prime} \mathrm{N}\right.$; $\left.11^{\circ} 17^{\prime} 58.53^{\prime \prime} \mathrm{E}\right)$; rel. 35: Obelisco di Sorio (Gambellara-M. Lessini-VI), 07-04-2010,

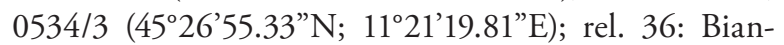
cara (Gambellara-M. Lessini-VI), 28-04-2011, 0534/1 (4527'40.97"; 1121'11.39”E); rel. 37: Sassonegro (Arquà Petrarca-Colli Euganei-PD), 08/04-19-05-2011, 0736/1

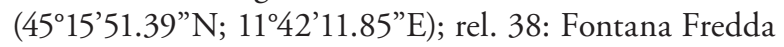
(Lazise-morainic hills-VR), 30/03-25/05/2012, 0530/1 $\left(45^{\circ} 27^{\prime} 44.66^{\prime \prime} \mathrm{N}\right.$; $\left.10^{\circ} 43^{\prime} 57.08^{\prime \prime} \mathrm{E}\right)$; rel. 39: Mascarpina (Custoza-morainic hills-VR), 30/03-25/05/2012, 0630/2 (4523’28.49”N; 1048'26.11”E); rel. 40: Canzago (Marano Valpolicella-M. Lessini-VR), 05/04-20/05/2011,

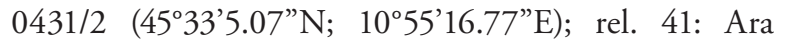
(Negrar-M. Lessini-VR), 05/04-20/05/2011, 0431/4 (4532'21.14”N; 1057’31.63”E); rel. 42: Piazzili (Cavaion Veronese-morainic hills-VR), 28/03-25/05/2012, 0430/4 (4530'59.91"N; 1047’23.29”E); rel. 43: Astore (Castiglione delle Stiviere-morainic hills-MN), 07/04/2017, (4523’40.75"N; 10³1'31.27”E); rel. 44: S. Lucia (Valeggio sul Mincio-morainic hills-VR), 30/03-

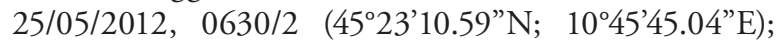
rel. 45: Colà (Lazise-morainic hills-VR), 30/0325/05/2012, 0530/2 (45⒉'12.78”N; $\left.10^{\circ} 45^{\prime} 8.49^{\prime \prime} \mathrm{E}\right)$; rel. 46: M. Cisa (Solighetto-foothills of Alps-TV), 18/04/2012, 0039/3 (4555'16.52”N; 12¹1'9.94”'E); rel. 47: Carbonarola (Grancona-Colli Berici-VI), 19/04/2015, $0534 / 4 \quad\left(45^{\circ} 24^{\prime} 6.80^{\prime \prime} \mathrm{N} ; \quad 11^{\circ} 27^{\prime} 0.63^{\prime \prime} \mathrm{E}\right)$; rel. 48: S. Benedetto (Marostica-foothills of Alps-VI),

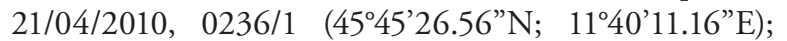
rel. 49: M. S. Lucia (Breganze-foothills of Alps-VI),

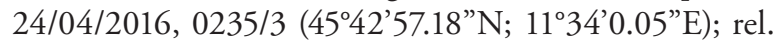
50: Casette (Este-Colli Euganei-PD), 08/04-19/05/2011,

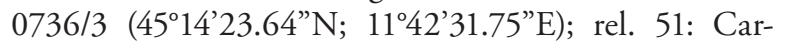


bonara (Rovolon-Colli Euganei-PD), 19/04/2016,

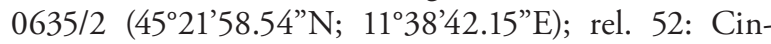
to Euganeo (Colli Euganei-PD), 29/04/2016, 0735/2 $\left(45^{\circ} 16^{\prime} 28.47^{\prime \prime N}\right.$; 11 $\left.39^{\circ} 6.28^{\prime \prime} \mathrm{E}\right)$; rel. 53: via Morette (Maser-Colli Asolani-TV), 25/04/2016, $0137 / 4$

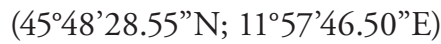

Appendix II: sporadic species of Table 2 Cerastio-Geranietum crepidetosum nemausensis; in parentheses relevé number with + value understood, except where otherwise stated.

Acer campestre (51); Achillea millefolium agg. (4, 27, 30, 46, 49: r); Aegopodium podagraria (7, 41); Agrimonia eupatoria (4: r; 24); Ajuga genevensis (3, 21, 40, 41); Ajuga reptans (7, 53); Allium ampeloprasum (37: 1; 50: 1); Allium longispathum (17, 50); Ammoides pusilla (8, 37); Arabidopsis thaliana (35: 1); Aristolochia clematitis (30); Arrhenatherum elatius (3: r; 46, 49); Asparagus acutifolius (6, 8, 39, 52); Brachypodium rupestre (5, 23, 46); Carex divulsa (15, 19, 31, 43); Carex pairae (4, 7, 15, 19, 26); Carex spicata (19, 44, 45, 46); Centaurea nigrescens/nigrescens (4: 2a; 6, 8, 9, 40, 41, 45, 46, 47); Cerastium glutinosum (15, 16, 18, 22, 23, 36, 42: r); Cerastium semidecandrum (20, 44: 1; 45: 1); Chondrilla juncea (22: 1; 27, 50); Cichorium intybus (21, 28, 29: r; 31, 39); Cirsium vulgare (24, 29, 31, 51: r); Clematis vitalba (6, 7, 10, 20, 24, 29, 47: r; 52); Cornus sanguinea (pl) (3); Dactylis glomerata $(4,7,24,32$, 48); Daucus carota (7, 8, 48, 50); Draba verna s.l. (24, 39: 1); Erigeron canadensis (18, 20, 37, 38, 39: 2a); Euphorbia cyparissias (8, 50); Ficaria verna s.l. (1, 2, 8); Galium album agg. (4, 8, 26, 40, 45, 46: 1); Galium verum (18, 30); Glechoma hederacea (4: 1; 6, 7, 10, 19: 2a; 26: 2b; 32, 40); Hedera helix (2, 3, 6: 1; 7, 10, 34, 47); Holosteum umbellatum/umbellatum (12: 1; 33); Hypochaeris radicata (49: r; 53); Lamium maculatum (26); Leontodon hispidus (20, 40: r; 53); Linaria vulgaris $(8,30)$; Lotus corniculatus (16, 41, 48); Medicago minima (11, 15: 2a; 16, 17, 22, 23, 38, 50, 51: 1; 52); Medicago sativa (19, 22); Mentha longifolia (1, 3, 6, 18: 1; 40, 46); Mentha suaveolens (20); Microthlaspi perfoliatum (32, 48); Muscari comosum (8: 1; 23, 27: r; 32, 34, 45); Myosotis ramosissima (23: 1; 33, 34: 1; 36, 48, 49: r; 53); Parietaria officinalis (10: 2a; 19, 25, 39, 45); Picris hieracioides (36, 51); Plantago media (4, 10, 41, 42: r); Poa angustifolia (16: 1; 23: 2b; 26: 1; 31, 48: 1); Poa bulbosa (18: 1; 35); Poa pratensis (4: 2a; 6: 1; 7: 1; 8, 15, 17, 24: 1; 25, 33: 1; 48: 2a); Polygonum aviculare agg. (10, 11, 12, 13, 16, 24, 25, 38, 42, 53: r); Potentilla argentea (33, 48: 1); Potentilla recta (22, 23); Poterium sanguisorba (23, 49, 50); Quercus pubescens ( $\mathrm{pl})$ (29: r; 30: r; 47); Ranunculus acris (7: 1); Ranunculus repens (7; 53);
Rosa canina s.l. (pl) (23, 24); Rumex crispus (4, 5, 6, 18, 51); Rumex obtusifolius (4, 7, 8, 9, 19: 1; 46); Salvia pratensis (23, 40, 41, 44, 46); Silene latifolia (12, 44, 46: 1); Silene vulgaris/vulgaris (3: r; 8, 22, 27, 40, 41, 48, 49, 51); Tragopogon dubius (17, 26, 27, 28, 32, 35: $\mathrm{r}$; 37, 38, 39, 50); Trifolium campestre (23, 30: 1; 34, 39: 1); Trifolium scabrum (18, 39); Ulmus minor (pl) (21, 27: r, 28: r; 29, 31: r); Urtica dioica (26, 44, 45, 46); Valerianella carinata (1: r; 3: r; 34: 1); Verbena officinalis (8, 13, 17: 1; 19; 31, 37, 38, 41); Vicia cracca (32: 2a; 33: 1); Vicia lathyroides $(24,35,36,48)$.

Appendix III: localities, dates, quadrants of relevés of Table 3.

Rel. 1: M. Paolina (Sarego-Colli Berici-VI), 02/04/2012, 0534/4; rel. 2: Grumale (Villaga-Colli Berici-VI), 04/04/2012, 0635/1; rel. 3: M. Paolina (Sarego-Colli Berici-VI), 02/04/2012, 0534/4; rel. 4: Sarego (Colli Berici-VI), 02/04/2012, 0534/3; rel. 5: Busa Geretta (Sarego-Colli Berici-VI), 04/04/2012, 0534/4; rel. 6: Preval (Collio Goriziano), 04/05/2012, 0047/1; rel. 7: Preval (Collio Goriziano), 11/05/2012, 0047/1; rel. 8: Preval (Collio Goriziano), 11/05/2012, 0047/1; rel. 9: Preval (Collio Goriziano), 15/06/2012, 0047/1; rel. 10: Preval (Collio Goriziano), 15/06/2012, 0047/1; rel. 11: Preval (Collio Goriziano), 15/06/2012, 0047/1; rel. 12: Preval (Collio Goriziano), 15/06/2012, 0047/1; rel. 13: Preval (Collio Goriziano), 15/06/2012, 0047/1; rel. 14: Preval (Collio Goriziano), 15/06/2012, 0047/1.

Appendix IV: sporadic species of Table 3; in parentheses relevé number with + value understood, except where otherwise stated.

Agrostis stolonifera (11); Ajuga reptans (13); Artemisia verlotiorum (2: r); Arrhenatherum elatius (6, 11); Bromus erectus (6); Calepina irregularis (5); Centaurea nigrescens/ nigrescens (9, 10); Cerastium brachypetalum/brachypetalum (3, 5); Dactylis glomerata (9); Galium mollugo s.l. (6); Hypochaeris radicata (1); Leucanthemum vulgare (5: r); Linaria vulgaris (14); Lychnis flos-cuculi (7); Lythrum salicaria (13); Microthlaspi perfoliatum (2: r); Muscari neglectum (3: r); Poterium sanguisorba (5); Quercus robur (pl) (12, 14); Ranunculus acris (8, 9); Ranunculus bulbosus (5); Rorippa sylvestris (9); Stachys palustris (13, 14); Trifolium campestre (11); Verbena officinalis (1: r; 13); Veronica chamaedrys (4). 
Table 1: Synoptic table of vineyard relevés from South-eastern (Ven: Veneto; Fri: Friuli Venezia Giulia; SLO: Slovenia) and Central (D: Germany; H: Hungary) Europe. D2: Oberdorfer (1983); D3: Orgis (1977). Included are companion species occurring at least once in $\geq$ II frequency class.

Tabela 1: Sinoptična tabela popisov vinogradov iz jugovzhodne (Ven: Benečija; Fri: Furlanija-Julijska krajina; SLO: Slovenija) in Srednje Evrope (D: Nemčija; H: Madžarska). D2: Oberdorfer (1983); D3: Orgis (1977). Prikazane so spremljevalne vrste, ki se pojavljajo enkrat, in $\mathrm{z}$ razredom frekvence $\geq$ II.

\begin{tabular}{lcccccc}
\hline geographical area & Ven & Fri+SLO & D1 & D2 & D3 & H \\
cluster (Figure 1) & A1 a & A1b & A2 & - & - & B \\
relevés $\left(\mathrm{n}^{\circ}\right)$ & 53 & 47 & 69 & 183 & 18 & 34 \\
\hline
\end{tabular}

sp. of Cerastio-Geranietum

Geranium dissectum $(\mathrm{Cl})$

Cerastium glomeratum $(\mathrm{Cl})$

Ornithogalum divergens $(\mathrm{Cl})$

Calepina irregularis

Crepis vesicarialtaraxacifolia $(\mathrm{Cl} ; \mathrm{d}$ subass. typicum)

Poa sylvicola

Cerastium brachypetalum/tenoreanum (d subass. typicum)

Lolium multiflorum

\begin{tabular}{|ll|}
\hline 57 & 60 \\
51 & 51 \\
62 & 32 \\
60 & 47 \\
23 & 66 \\
45 & 43 \\
13 & 57 \\
23 & 40 \\
\hline
\end{tabular}

sp. of Cerastio-Geranietum subass. crepidetosum nemausensis

Crepis sanctalnemausensis $(\mathrm{Cl})$

Geranium molle $(\mathrm{Cl})$

Hordeum murinum s.l. (Cl)

Cynodon dactylon $(\mathrm{O})$

Cardamine hirsuta $(\mathrm{Cl})$

Rumex pulcher/pulcher

Malva sylvestris

Torilis nodosa/nodosa $(\mathrm{Cl})$

Avena barbata $(\mathrm{Cl})$

Lactuca saligna $(\mathrm{Cl})$

Ranunculus parviflorus $(\mathrm{Cl})$

\begin{tabular}{|c|c|}
\hline 92 & $\cdot$ \\
89 & 13 \\
72 & 2 \\
70 & 17 \\
62 & 17 \\
55 & $\cdot$ \\
47 & 2 \\
38 & $\cdot$ \\
34 & $\cdot$ \\
26 & $\cdot$ \\
25 & 2 \\
& \\
9 & 40 \\
$\cdot$ & 34 \\
$\cdot$ & 19 \\
. & 17 \\
\hline
\end{tabular}

sp. of Cerastio-Geranietum subass. typicum

Rumex crispus

Alopecurus myosuroides $(\mathrm{Cl})$

Trifolium incarnatum/molinerii

Anthemis arvensis $(\mathrm{Cl})$

sp. of Geranio-Allietum

Allium vineale $(\mathrm{Cl}$; p.max.p. $)+$ A. oleraceum $(\mathrm{Cl})$

Muscari neglectum

$\begin{array}{cccccc}45 & 47 & 81 & 84 & \text { IV } & 6 \\ 64 & 43 & 45 & 51 & \text { II } & 38 \\ . & . & 67 & 23 & . & 65 \\ 9 & . & 32 & 16 & \text { II } & 24 \\ 49 & 13 & 46 & 21 & . & . \\ 6 & 9 & 52 & 2 & . & . \\ 6 & 4 & 26 & . & . & . \\ . & . & 14 & 11 & \text { III } & . \\ . & . & 3 & . & . & 18 \\ . & . & 4 & . & \text { III } & . \\ . & . & 29 & . & . & . \\ . & . & 9 & . & . & .\end{array}$




\section{geographical area}

Ven Fri+SLO D1

Calendula arvensis (all)

Allium nigrum $(\mathrm{Cl})$

sp. of cluster B

Holosteum umbellatum

Viola arvensis $(\mathrm{Cl})$

Vicia grandiflora

Androsace maxima $(\mathrm{Cl})$

$\begin{array}{lll}\cdot & \cdot & 4 \\ . & \cdot & 3\end{array}$

sp. of Veronico-Euphorbion

\begin{tabular}{|c|c|c|c|c|}
\hline 4 & & 3 & II & 62 \\
\hline 8 & 6 & . & 4 & 41 \\
\hline & & . & . & 38 \\
\hline & & & . & 18 \\
\hline
\end{tabular}

Euphorbia helioscopia

Fumaria officinalis

Mercurialis annua

Veronica polita

Thlaspi arvense

Euphorbia peplus

sp. of Solano-Polygonetalia

Lamium purpureum

Sonchus oleraceus

Lamium amplexicaule

Sonchus asper

Erodium cicutarium

Fallopia convolvulus

Chenopodium album

Persicaria maculosa

sp. of Stellarietea mediae

Stellaria medialmedia

Veronica persica

Convolvulus arvensis

Senecio vulgaris

Capsella bursa-pastoris

Veronica hederifolia

Cirsium arvense

Anisantha sterilis

Veronica arvensis

Geranium pusillum

Malva neglecta

Vicia sativa agg.

Papaver rhoeas

Anagallis arvensis

Sinapis arvensis

Ervilia hirsuta

Lepidium draba

Sisymbrium officinale

Valerianella locusta

Geranium columbinum

Crepis pulchra

Myosotis arvensis

Sherardia arvensis

Senecio leucanthemifolius/vernalis

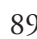

45

26

$\begin{array}{cccccc}89 & 79 & 32 & 78 & \text { II } & 15 \\ 45 & 23 & 29 & 54 & \text { III } & 12 \\ 26 & 28 & 43 & 52 & . & . \\ . & . & 28 & 58 & . & 62 \\ . & 4 & . & 6 & . & . \\ . & . & . & 33 & . & .\end{array}$

$\begin{array}{cccccc}64 & 30 & 78 & 89 & \text { II } & 74 \\ 60 & 45 & 29 & 91 & \text { I } & 6 \\ 57 & 21 & 10 & 30 & \text { I } & 74 \\ 45 & 34 & 28 & 81 & \text { I } & . \\ 19 & 38 & 14 & 29 & . & 44 \\ 2 & 21 & 7 & 3 & \text { II } & . \\ 9 & 9 & 12 & . & \text { II } & . \\ . & 4 & 1 & . & . & .\end{array}$

$\begin{array}{cccccc}94 & 98 & 74 & 80 & \text { IV } & 85 \\ 98 & 98 & 72 & 64 & \text { I } & 50 \\ 87 & 70 & 84 & 41 & \text { V } & 47 \\ 79 & 81 & 33 & 84 & \text { II } & 38 \\ 81 & 74 & 23 & 25 & \text { I } & 74 \\ 30 & 34 & 84 & 50 & \text { II } & 56 \\ 34 & 68 & 36 & 56 & \text { II } & 3 \\ 57 & 45 & 70 & 20 & . & 38 \\ 62 & 23 & 20 & 13 & . & 47 \\ 28 & 2 & 10 & 11 & . & 68 \\ 15 & 4 & 23 & 17 & \text { I } & . \\ 43 & 72 & 32 & . & . & 35 \\ 21 & 23 & 10 & 9 & . & . \\ 2 & 17 & 3 & 16 & . & . \\ . & 4 & 6 & 20 & \text { I } & . \\ 4 & 4 & 13 & . & . & 6 \\ . & 6 & 1 & . & \text { I } & 12 \\ 2 & 4 & 1 & 1 & . & . \\ 23 & 11 & . & . & . & 35 \\ 4 & 17 & . & 15 & . & . \\ 4 & . & 10 & . & . & 6 \\ 6 & 11 & . & 3 & . & . \\ 6 & 2 & . & 2 & . & . \\ . & . & 49 & . & . & 9\end{array}$


geographical area

Diplotaxis muralis

Anisantha madritensis

Papaver dubium

Buglossoides arvensis

Erodium ciconium

Delphinium consolida

Scandix pecten-veneris/pecten-veneris

Ranunculus arvensis

Aphanes arvensis

Capsella rubella

Malva setigera

Urtica urens

Oxalis stricta

Erigeron sumatrensis

Torilis arvensis

Diplotaxis tenuifolia

Sorghum halepense

Crepis vesicarialvesicaria

Raphanus raphanistrum

Oxalis dillenii

Vicia hybrida

Crepis foetida

Avena fatualfatua

Legousia hybrida

Loncomelos brevistylus

Medicago orbicularis

Veronica triphyllos

Euphorbia exigua

Legousia speculum-veneris

Chaenorhinum minus/minus

Papaver dubium/confine

Medicago arabica

Bromus arvensis

Anisantha diandra

Medicago polymorpha

Oxalis corniculata

Papaver argemone

Aethusa cynapium

Trifolium arvense

Lathyrus sphaericus

Fumaria vaillantii

Adonis aestivalis

Veronica agrestis

sp. of Artemisietea vulgaris

Elymus repens

Sonchus arvensis

Erigeron annuus

Rumex obtusifolius s.1.

Artemisia verlotiorum

4

17

17

8

8

8

4

4

2

$\begin{array}{llllll}\text { Ven } & \text { Fri+SLO } & \text { D1 } & \text { D2 } & \text { D3 } & \text { H }\end{array}$

23

. 6

(1)




\section{geographical area}

Ven Fri+SLO D1

D2

sp. of Cynosurion cristati or with stolons

Lolium perenne $(\mathrm{Cy})$

Trifolium repens $(\mathrm{Cy})$

Potentilla reptans

Bellis perennis (Cy)

$\begin{array}{cccccc}79 & 2 & 33 & 4 & . & 3 \\ 70 & 11 & 1 & . & . & 6 \\ 28 & 13 & 3 & . & . & . \\ 60 & 2 & . & . & . & .\end{array}$

other sp. of Molinio-Arrhenatheretea

Taraxacum sect. Ruderalia

Ranunculus repens

Plantago lanceolata

Trifolium pratense/pratense

Tragopogon pratensis

$\begin{array}{cccccc}92 & 72 & 87 & 91 & \text { IV } & 74 \\ 4 & 9 & 7 & 26 & . & . \\ 64 & 15 & . & . & . & . \\ 32 & 9 & . & . & . & . \\ . & 2 & . & . & . & 32\end{array}$

other

Lactuca sativa/serriola

Poa annua

Galium aparine

Medicago lupulina

Arenaria serpyllifolia

Polygonum aviculare agg.

Bromus hordeaceus s.l.

Microthlaspi perfoliatum

Urtica dioica

Plantago major

Cerastium brachypetalum/brachypetalum

Ranunculus bulbosus

Hedera helix

$\begin{array}{cccccc}79 & 51 & 58 & 22 & \text { I } & 38 \\ 34 & 32 & 48 & 22 & \text { I } & 12 \\ 21 & 6 & 59 & 10 & \text { I } & 18 \\ 21 & 2 & 3 & 8 & . & 3 \\ 42 & 17 & . & 15 & . & 50 \\ 19 & 2 & 26 & . & \text { I } & \cdot \\ 21 & 6 & 1 & . & . & 3 \\ 4 & . & 1 & . & \text { III } & 15 \\ 8 & 2 & 23 & . & . & . \\ 21 & 2 & 3 & . & . & . \\ 49 & . & . & . & . & 24 \\ 32 & 26 & . & . & . & . \\ 13 & . & 25 & . & . & . \\ 13 & 21 & . & . & . & .\end{array}$


Table 2: Analytical table of vineyard vegetation from Veneto (North-eastern Italy). C.A.: Colli Asolani; C.B.: Colli Berici; C.E.: Colli Eugaenei; G.m.: Garda moraines; L.: Monti Lessini; P.: Prealps. Included are companion species occurring in $\geq$ II frequency class.

\begin{tabular}{|c|c|c|c|c|c|c|c|c|c|c|c|c|c|c|c|c|c|c|c|c|c|c|c|}
\hline relevé $\mathrm{n}^{\circ}$ & 1 & 2 & 3 & 4 & 5 & 6 & 7 & 8 & 9 & 10 & 11 & 12 & 13 & 14 & 15 & 16 & 17 & 18 & 19 & 20 & 21 & 22 & 23 \\
\hline elevation a.s.l. (m) & 50 & 70 & 275 & 260 & 60 & 130 & 80 & 75 & 105 & 230 & 145 & 140 & 70 & 100 & 120 & 170 & 90 & 230 & 65 & 110 & 60 & 90 & 175 \\
\hline number of species & 33 & 31 & 45 & 46 & 41 & 43 & 47 & 54 & 38 & 44 & 33 & 36 & 36 & 29 & 45 & 45 & 44 & 53 & 46 & 41 & 39 & 38 & 50 \\
\hline geographical area & $\stackrel{\varphi}{\dot{v}}$ & $\dot{\sim}$ & $\dot{1}$ & $\dot{ن}$ & $\stackrel{\text { ن }}{ن}$ & $\dot{-1}$ & $\dot{\varphi}$ & نَ & $\dot{v}$ & $\dot{\sim}$ & $\dot{v}$ & ن & $\dot{ن}$ & نَ & $\stackrel{\varphi}{\dot{0}}$ & $\dot{ن}$ & $\dot{-1}$ & $\dot{-1}$ & $\stackrel{\varphi}{\dot{U}}$ & $\underset{ن}{\dot{\Xi}}$ & ند & $\dot{ن}$ & $\dot{ن}$ \\
\hline sp. of Cerastio-Geranietun & & & & & & & & & & & & & & & & & & & & & & & \\
\hline Ornithogalum divergens & 1 & + & $\mathrm{r}$ & + & . & + & . & + & + & + & . & $2 \mathrm{a}$ & + & . & + & 1 & 1 & 1 & + & $\mathrm{r}$ & $\mathrm{r}$ & + & + \\
\hline Calepina irregularis & + & 3 & + & + & + & 1 & $2 b$ & + & $2 \mathrm{a}$ & + & . & . & + & + & . & . & . & + & . & + & + & . & $2 \mathrm{a}$ \\
\hline Geranium dissectum & + & . & + & $2 \mathrm{a}$ & 3 & 1 & $2 \mathrm{a}$ & . & + & . & + & $r$ & . & + & . & . & . & . & . & + & $2 b$ & + & $2 a$ \\
\hline Cerastium glomeratum & . & . & + & + & + & . & . & . & + & + & + & + & 1 & + & + & + & + & 1 & + & . & $2 \mathrm{a}$ & . & . \\
\hline Poa sylvicola & . & + & . & . & 1 & 1 & 1 & + & $2 \mathrm{a}$ & 1 & . & . & . & . & . & . & + & + & $2 a$ & + & . & . & . \\
\hline Crepis vesicarialtaraxacifolia & . & . & . & . & $2 \mathrm{a}$ & . & + & + & . & . & . & . & . & . & . & + & + & . & . & . & . & + & $2 \mathrm{a}$ \\
\hline Lolium multiflorum & . & . & . & . & . & . & 1 & . & . & . & . & . & . & . & . & . & . & . & + & 1 & $2 \mathrm{a}$ & . & . \\
\hline Cerastium brachypetalum/tenoreanum & . & . & + & 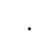 & & 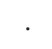 & - & 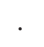 & . & 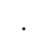 & 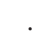 & 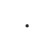 & & & . & + & - & & & . & . & + & 1 \\
\hline
\end{tabular}

sp. of Cerastio-Geranietum subass crepidetosum nemausensis

Crepis sancta/nemausensis $\quad+2 \mathrm{a} 3+2 \mathrm{~b} 2 \mathrm{a}+++2 \mathrm{a}+2 \mathrm{a} 2 \mathrm{~b} 42 \mathrm{~b} 2 \mathrm{~b} 2 \mathrm{~b} 2 \mathrm{~b} 32 \mathrm{a} 2 \mathrm{a} 2 \mathrm{~b} 2 \mathrm{a}$

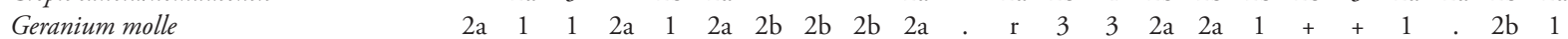

Hordeum murinum/leporinum $\quad 2 \mathrm{a}+\cdot 1+2 \mathrm{a} \cdot 2 \mathrm{a} 2 \mathrm{~b} 2 \mathrm{a} \cdot 2 \mathrm{a} 2 \mathrm{a} 2 \mathrm{a}+1+2 \mathrm{a} 2 \mathrm{a}+2 \mathrm{a}+$

Cynodon dactylon

Cardamine hirsuta

Rumex pulcher/pulcher

Malva sylvestris

Torilis nodosa/nodosa

Avena barbata

Lactuca saligna

Ranunculus parviflorus

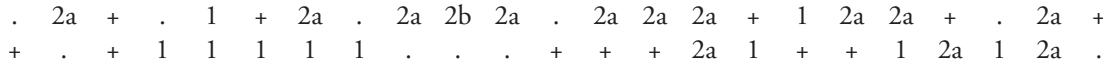

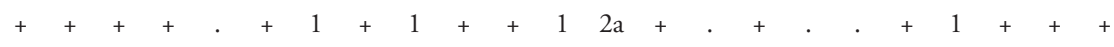

sp. of Veronico-Euphorbion

Euphorbia helioscopia

Fumaria officinalis s.l.

Mercurialis annua

sp. of Solano-Polygonetalia

Lamium purpureum

Sonchus oleraceus

Lamium amplexicaule

Geranium rotundifolium

Sonchus asper

Erodium cicutarium

Gagea villosa

Chenopodium album $(\mathrm{pl})$

Fallopia convolvulus

sp. of Stellarietea mediae

Veronica persica

Stellaria media

Convolvulus arvensis

Capsella bursa-pastoris

Senecio vulgaris

Veronica arvensis

Anisantha sterilis

Allium vineale

Vicia sativa agg. (V.angustifolia p.max.p)

Cirsium arvense

Veronica hederifolia

Geranium pusillum

Erigeron sumatrensis

Valerianella locusta

Papaver rhoeas

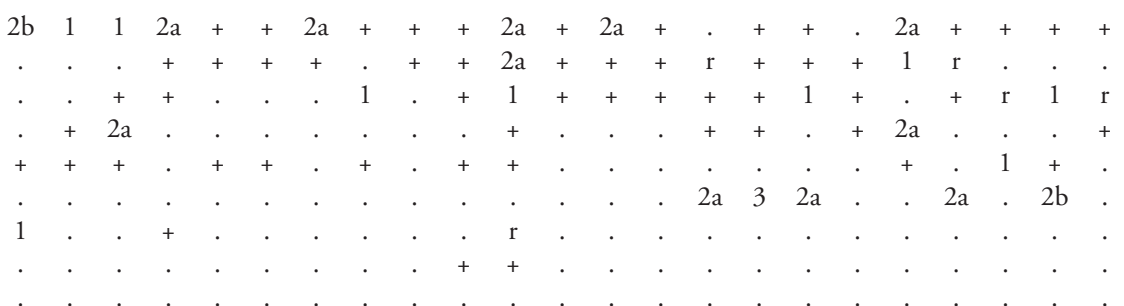


Tabela 2: Analitična tabela vegetacije vinogradov v Benečiji (severovzhodna Italija). C.A.: Colli Asolani; C.B.: Colli Berici; C.E.: Colli Eugaenei; G.m.: morena Garda; L.: Monti Lessini; P.: Predalpe. Prikazane so spremljevalne vrste, ki se pojavljajo enkrat, in z razredom frekvence $\geq$ II.

$\begin{array}{lllllllllllllllllllllllllllllllll}24 & 25 & 26 & 27 & 28 & 29 & 30 & 31 & 32 & 33 & 34 & 35 & 36 & 37 & 38 & 39 & 40 & 41 & 42 & 43 & 44 & 45 & 46 & 47 & 48 & 49 & 50 & 51 & 52 & 53 & \text { pr. } & \text { fr. }\end{array}$

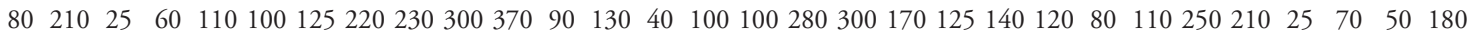
$\begin{array}{llllllllllllllllllllllllllllll}37 & 33 & 50 & 52 & 42 & 37 & 46 & 44 & 46 & 31 & 48 & 31 & 33 & 39 & 35 & 35 & 31 & 43 & 37 & 32 & 36 & 35 & 40 & 35 & 47 & 30 & 41 & 45 & 38 & 29\end{array}$

\begin{tabular}{|c|c|c|c|c|c|c|c|c|c|c|c|c|c|c|c|c|c|c|c|c|c|c|c|c|c|c|c|c|c|c|c|}
\hline$\dot{H}$ & $\dot{1}$ & ضُ & $\dot{ن}$ & ن & نُ & ن & 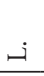 & نै & $\dot{-1}$ & $\dot{-1}$ & ــ & $\dot{~}$ & نُ & હ் & હ્ધું & $\dot{-i}$ & $\dot{-1}$ & غ્ & غ્ધ் & غ્દ & घ્ં & Ai & 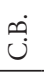 & ai & Si & نُ & نُ & نَّ & ذ্் & & \\
\hline & + & + & + & . & + & $\cdot$ & . & & . & . & & + & + & & . & + & + & 1 & + & 1 & & & & & & $2 a$ & 1 & & & $\begin{array}{l}\text { pr. } \\
33\end{array}$ & \\
\hline 1 & $2 \mathrm{a}$ & 1 & + & . & + & + & . & . & . & 3 & + & $\cdot$ & . & $2 a$ & & - & . & + & & 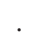 & + & + & + & . & + & . & + & & & 32 & \\
\hline+ & 1 & 1 & . & + & $2 b$ & + & . & . & . & 1 & . & + & 1 & & . & . & . & $\mathrm{r}$ & $2 a$ & & . & & + & + & & & & . & $2 b$ & 30 & \\
\hline . & . & + & 1 & + & $2 \mathrm{a}$ & + & . & . & . & . & 1 & 1 & . & & . & . & . & . & & . & & & . & $2 \mathrm{a}$ & & 1 & + & + & $2 b$ & 27 & \\
\hline 1 & + & . & + & . & . & . & . & . & . & . & . & . & + & 1 & . & . & $2 \mathrm{a}$ & + & 1 & . & + & + & + & . & + & . & . & . & $2 \mathrm{a}$ & 24 & \\
\hline+ & . & . & . & . & . & . & . & + & . & + & . & . & . & & . & . & . & . & $\cdot$ & . & . & + & . & . & $\mathrm{r}$ & . & $\cdot$ & . & $\cdot$ & 12 & \\
\hline & . & . & . & . & . & 1 & . & . & . & + & . & . & & & . & . & . & $2 b$ & & $2 a$ & + & + & & & & . & + & . & + & 12 & \\
\hline & . & . & . & . & & . & & . & . & + & & & & & & + & + & & & & & & & & & & & & & 7 & \\
\hline
\end{tabular}

\begin{tabular}{|c|c|c|c|c|c|c|c|c|c|c|c|c|c|c|c|c|c|c|c|c|c|c|c|c|c|c|c|c|c|}
\hline & $2 a$ & 3 & 3 & $2 b$ & $2 b$ & 3 & $2 a$ & 3 & $2 a$ & $2 b$ & $2 a$ & + & $2 b$ & & $2 b$ & $2 b$ & $2 a$ & 1 & + & $2 \mathrm{a}$ & & 1 & & & $2 \mathrm{~b}$ & 3 & $2 \mathrm{~b}$ & & 49 \\
\hline & $2 a$ & 1 & . & . & $2 b$ & $2 a$ & + & $2 a$ & $2 b$ & + & + & 3 & 3 & 1 & $2 b$ & $2 b$ & 3 & 3 & + & $2 b$ & $2 a$ & $2 a$ & + & $2 b$ & + & & . & $2 a$ & 47 \\
\hline & $2 b$ & + & + & 1 & & 1 & 1 & $2 a$ & + & + & $2 b$ & 1 & $2 b$ & & + & 1 & $2 \mathrm{a}$ & & $2 b$ & & & $2 b$ & & 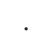 & & $\mathrm{r}$ & + & & 38 \\
\hline & 1 & + & 1 & 1 & $2 b$ & 1 & $2 \mathrm{a}$ & 1 & & & . & $2 \mathrm{a}$ & + & $2 b$ & & & . & + & 1 & & & & 1 & $2 a$ & 1 & $2 a$ & & & 37 \\
\hline . & + & + & 1 & . & 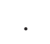 & + & 1 & 1 & + & 1 & . & 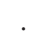 & & . & & & & . & & & . & & & . & & & & + & 33 \\
\hline $2 b$ & + & + & + & $\mathrm{r}$ & . & . & . & . & . & + & + & 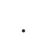 & & & & & $\mathrm{r}$ & + & + & $\cdot$ & . & $2 a$ & + & . & + & . & & . & 29 \\
\hline & + & . & . & . & + & . & + & 1 & 1 & $2 b$ & . & $2 \mathrm{a}$ & & + & . & . & + & $2 a$ & + & + & . & . & + & + & . & . & & . & 25 \\
\hline & . & . & + & $2 \mathrm{a}$ & + & $2 b$ & + & . & . & . & . & . & & . & $\mathrm{r}$ & + & . & . & . & . & . & . & 1 & & & + & & & 20 \\
\hline & 1 & + & 1 & 1 & + & 1 & $2 \mathrm{a}$ & . & . & & . & . & & + & . & & . & & & & & & . & + & + & . & & . & 18 \\
\hline & . & . & + & . & & + & + & & + & & . & & & & + & & & & & & · & & & & & & & & 14 \\
\hline & & & 1 & 1 & + & & & & 1 & & $2 \mathrm{a}$ & & & & & & & 1 & & & & $2 \mathrm{a}$ & & & & + & & & 13 \\
\hline
\end{tabular}
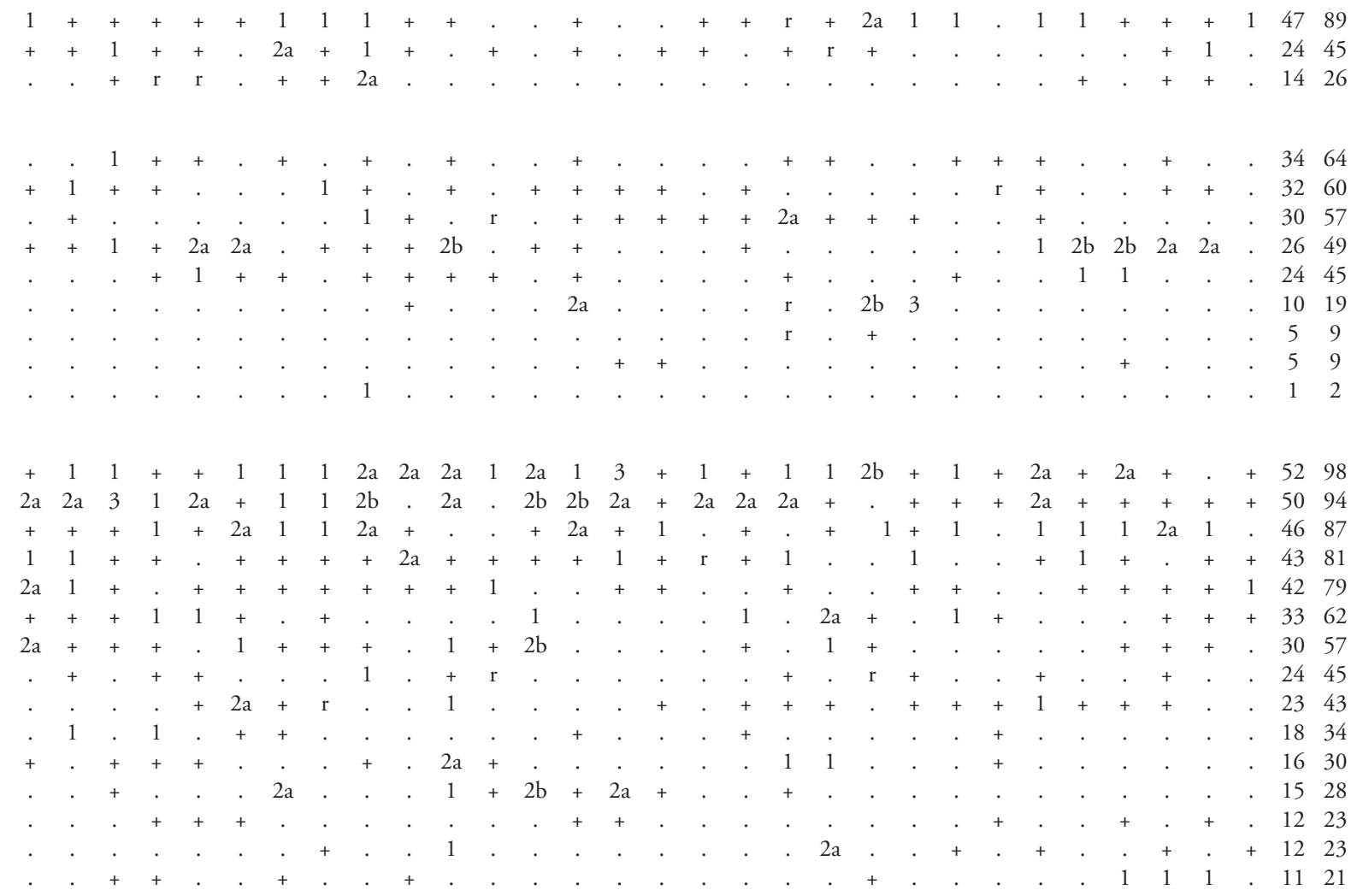


\section{relevé $\mathrm{n}^{\circ}$}

Anisantha madritensis

Papaver dubium

Diplotaxis tenuifolia

Malva neglecta

Crepis vesicarialvesicaria

Sorghum halepense

Oxalis dillenii

Vicia hybrida

Avena fatualfatua

Buglossoides arvensis

Erodium ciconium

Legousia hybrida

Loncomelos brevistylus

Medicago orbicularis

Scandix pecten-veneris/pecten-veneris

Viola arvensis

Medicago arabica

Myosotis arvensis

Sherardia arvensis

Aphanes arvensis

Bromus arvensis

Anisantha diandra

Capsella rubella

Crepis pulchra

Diplotaxis muralis

Geranium columbinum

Medicago polymorpha

Oxalis corniculata

Papaver argemone

Valerianella turgida

Ervilia hirsuta

Lathyrus sphaericus

Anagallis arvensis

Oxalis stricta

Sisymbrium officinale

sp. of Artemisietea vulgaris

Erigeron annuus

Elymus repens

Artemisia verlotiorum

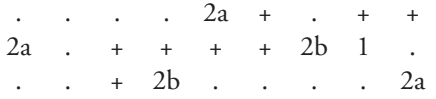

sp. of Cynosurion cristati or with stolons

Lolium perenne $(\mathrm{Cy})$

Trifolium repens $(\mathrm{Cy})$

Bellis perennis $(\mathrm{Cy})$

Potentilla reptans

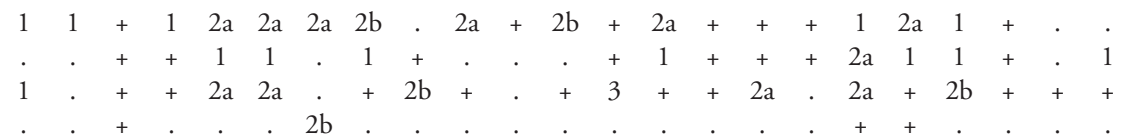

other sp. of Molinio-Arrhenatheretea

Taraxacum sect. Ruderalia

Plantago lanceolata

Trifolium pratense/pratense

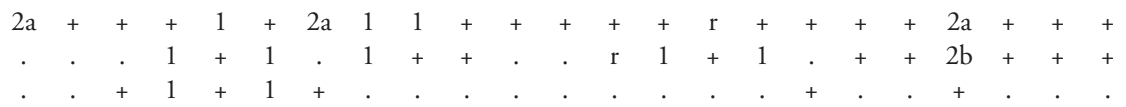

others

Lactuca sativalserriola

Muscari neglectum

Cerastium brachypetalum/brachypetalum

Arenaria serpyllifolia

Poa annua

Ranunculus bulbosus

Bromus hordeaceus s.l.

Galium aparine

Medicago lupulina

Plantago major

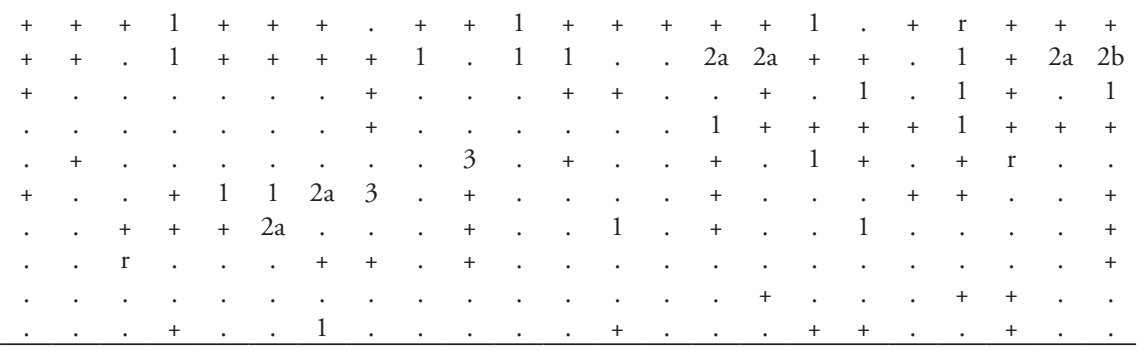



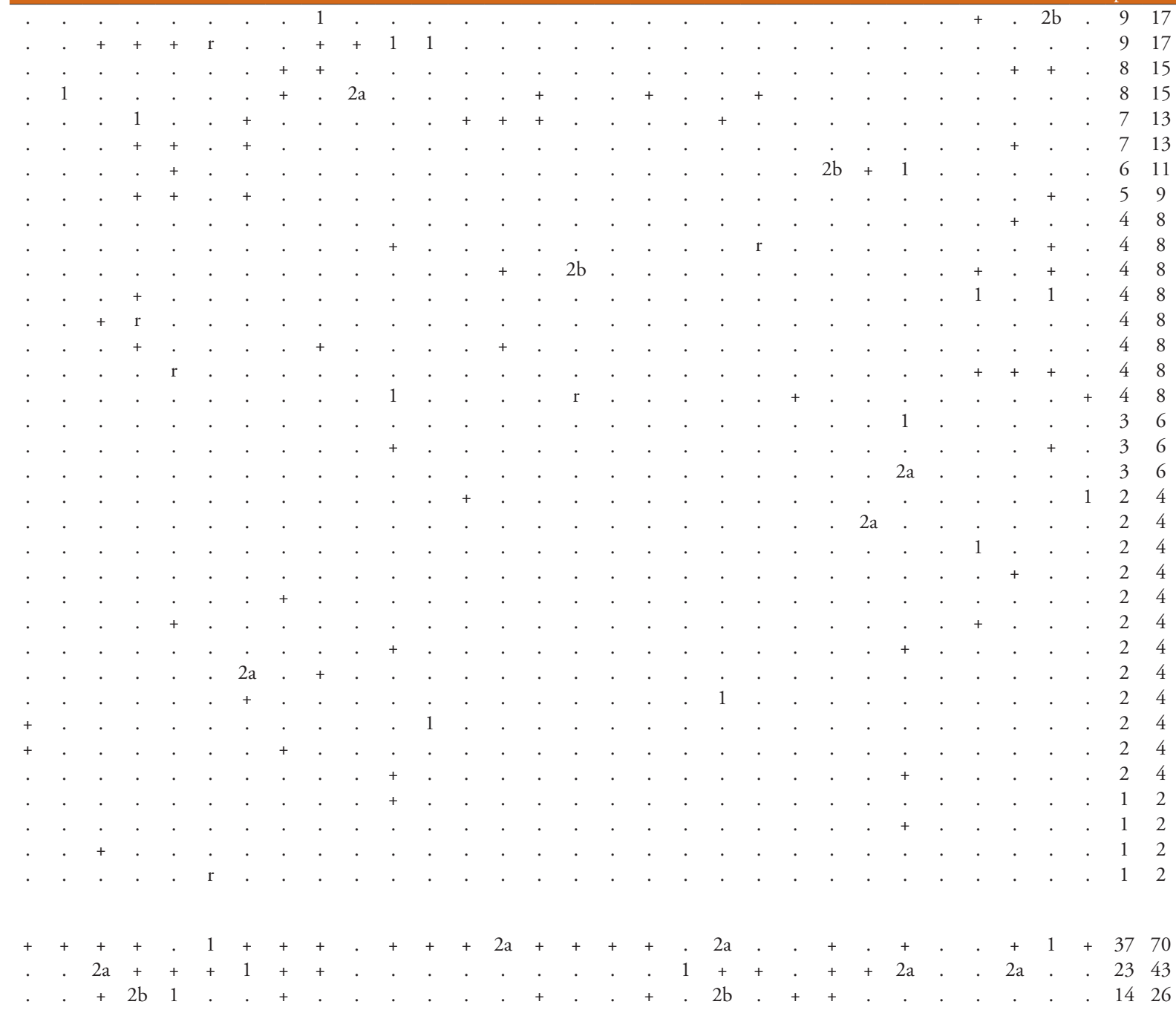

$$
\begin{aligned}
& 2 \mathrm{a}+2 \mathrm{a}+12 \mathrm{a} \cdot++1+3 \cdot 1+12 \mathrm{~b} 32 \mathrm{a}++2 \mathrm{~b}+. .+42 .+49
\end{aligned}
$$

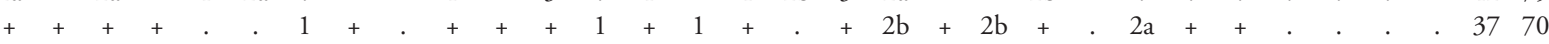

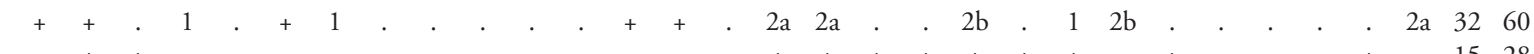

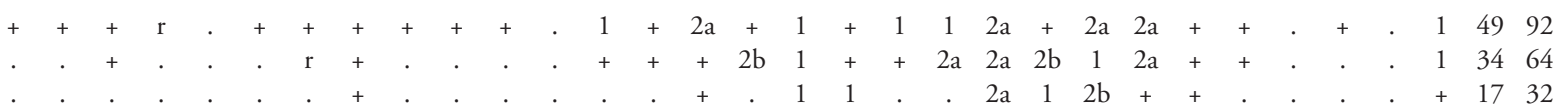

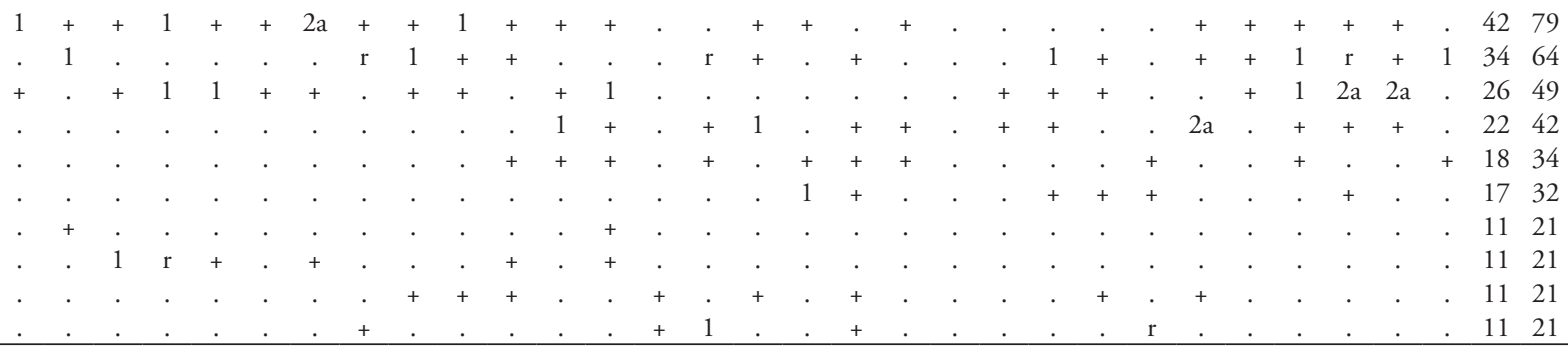


Table 3: Analytical table of vineyards from North-eastern Italy sown with grasses. Included are companion species occurring at least once in $\geq$ II frequency class. For abbreviations see Table 1 .

Tabela 3: Analitična tabela vegetacije vinogradov iz severnovzhodne Italije s sejanimi travami. . Prikazane so spremljevalne vrste, ki se pojavljajo enkrat, in $\mathrm{z}$ razredom frekvence $\geq$ II. Okrajšave so prikazane v Tabeli 1.

\begin{tabular}{|c|c|c|c|c|c|c|c|c|c|c|c|c|c|c|c|c|}
\hline relevé $n^{\circ}$ & 1 & 2 & 3 & 4 & 5 & 6 & 7 & 8 & 9 & 10 & 11 & 12 & 13 & 14 & & \\
\hline number of species & 19 & 17 & 24 & 21 & 26 & 20 & 21 & 18 & 30 & 21 & 19 & 19 & 26 & 20 & & \\
\hline geographical area & Ven & Ven & Ven & Ven & Ven & Fri & Fri & Fri & Fri & Fri & Fri & Fri & Fri & Fri & & \\
\hline \multicolumn{17}{|l|}{ sowed species } \\
\hline Lolium perenne & . & . & + & 4 & $2 b$ & + & 2 & 1 & 3 & 2 & + & 1 & 3 & 1 & 12 & 86 \\
\hline Lolium arundinaceum $\mathrm{cv}$. & 3 & 4 & 3 & . & 4 & . & . & . & + & . & . & . & . & . & 5 & 36 \\
\hline \multicolumn{17}{|l|}{ sp. of Veronico-Euphorbion } \\
\hline Euphorbia helioscopia & . & + & . & 1 & + & . & + & . & . & . & . & . & . & . & 4 & 29 \\
\hline Fumaria officinalis s.l. & . & . & $\mathrm{r}$ & $\mathrm{r}$ & $\cdot$ & . & . & . & . & . & . & . & + & . & 3 & 21 \\
\hline \multicolumn{17}{|l|}{ sp. of Solano-Polygonetalia } \\
\hline Sonchus asper & 1 & . & . & $\mathrm{r}$ & . & + & + & 1 & . & . & . & . & . & . & 5 & 36 \\
\hline Sonchus oleraceus & . & . & . & . & $\mathrm{r}$ & . & . & . & + & + & . & . & + & + & 5 & 36 \\
\hline Chenopodium album (pl) & 1 & . & . & + & . & . & . & . & . & . & . & . & + & + & 4 & 29 \\
\hline Lamium purpureum & . & . & $\mathrm{r}$ & + & $\mathrm{r}$ & . & . & . & . & . & . & . & . & . & 3 & 21 \\
\hline Cynodon dactylon & . & + & . & . & . & . & . & . & + & . & . & . & . & . & 2 & 14 \\
\hline Geranium rotundifolium & . & . & . & 1 & . & . & . & . & . & . & . & . & . & . & 1 & 7 \\
\hline
\end{tabular}

sp. of Stellarietea mediae

Veronica persica

Convolvulus arvensis

Stellaria medialmedia

Capsella bursa-pastoris

Cerastium glomeratum

Crepis vesicaria s.l.

Geranium dissectum

Veronica arvensis

Crepis sancta/nemausensis

Senecio vulgaris

Cardamine hirsuta

Anagallis arvensis

Cirsium arvense

Sorghum halepense

Veronica hederifolia

Vicia sativa agg.

Avena fatua

Geranium molle

Hordeum leporinum

Persicaria maculosa

Anisantha sterilis

Diplotaxis tenuifolia

Oxalis stricta

Papaver rhoeas

Ranunculus parviflorus

Ornithogalum divergens 


\begin{tabular}{|c|c|c|c|c|c|c|c|c|c|c|c|c|c|c|c|c|}
\hline relevé $\mathrm{n}^{\circ}$ & 1 & 2 & 3 & 4 & 5 & 6 & 7 & 8 & 9 & 10 & 11 & 12 & 13 & 14 & pr. & fr. \\
\hline Chenopodium polyspermum & . & . & . & . & . & . & . & . & . & + & . & . & . & . & 1 & 7 \\
\hline Helminthotheca echioides & . & . & . & . & + & . & . & . & . & . & . & . & . & . & 1 & 7 \\
\hline \multicolumn{17}{|c|}{ other sp. of Molinio-Arrhenatheretea } \\
\hline Taraxacum sect. Ruderalia & 1 & + & + & $\mathrm{r}$ & $\mathrm{r}$ & 3 & 1 & + & + & + & 3 & 1 & + & + & 14 & 100 \\
\hline Trifolium repens & + & + & + & . & 1 & 1 & 1 & + & 1 & 1 & + & 2 & 1 & 1 & 13 & 93 \\
\hline Plantago lanceolata & . & + & . & . & $\mathrm{r}$ & . & + & + & 1 & + & + & + & + & 1 & 10 & 71 \\
\hline Lolium pratense & . & . & + & . & . & 3 & 1 & + & + & + & . & . & + & . & 7 & 50 \\
\hline Poa sylvicola & . & . & . & . & . & 1 & + & 1 & + & . & . & . & + & . & 5 & 36 \\
\hline Rumex crispus & . & . & + & . & . & . & + & . & + & + & . & . & 1 & . & 5 & 36 \\
\hline Lotus corniculatus & . & . & . & . & + & . & . & . & + & + & . & . & + & 1 & 5 & 36 \\
\hline Bellis perennis & . & . & . & . & . & + & + & + & + & . & . & . & . & . & 4 & 29 \\
\hline Vicia cracca & . & . & . & . & . & . & . & . & . & + & + & 1 & . & + & 4 & 29 \\
\hline Trifolium pratenselpratense & . & . & . & . & . & 2 & 1 & . & + & . & . & . & . & . & 3 & 21 \\
\hline Ranunculus repens & . & . & . & . & . & 1 & 1 & . & . & . & . & . & 1 & . & 3 & 21 \\
\hline \multicolumn{17}{|l|}{ others } \\
\hline Erigeron annuus & + & . & . & . & . & + & 1 & 2 & + & . & + & + & + & + & 9 & 64 \\
\hline Polygonum aviculare agg. & + & + & + & . & . & . & . & . & . & + & . & . & + & + & 6 & 43 \\
\hline Medicago lupulina & . & . & . & . & . & + & + & . & + & + & . & 1 & . & . & 5 & 36 \\
\hline Lactuca sativa/serriola & + & $\mathrm{r}$ & . & . & + & . & . & . & + & . & . & . & . & . & 4 & 29 \\
\hline Plantago major & . & . & . & . & . & . & . & . & + & . & . & + & + & + & 4 & 29 \\
\hline Bromus hordeaceus s.l. & . & . & . & . & $\mathrm{r}$ & . & . & . & . & . & + & + & . & . & 3 & 21 \\
\hline Equisetum arvense & . & . & . & . & . & + & . & + & . & . & . & . & . & + & 3 & 21 \\
\hline Cichorium intybus & . & . & . & . & . & . & . & . & + & + & + & . & . & . & 3 & 21 \\
\hline
\end{tabular}

Table 4: Cover range, frequency and percentage frequency of species occurring in 40 relevés from Veneto in summer-autumn phenophase and absent or little represented in spring one. Included are companion species occurring in $\geq$ II frequency class.

Tabela 4: Razpon pokrovnosti, frekvenca in frekvenca vrst v odstotkih v 40 popisih poletno - jesenske fenofaze iz Benečije in vrste, ki so odsotne ali redke v spomladanski fenofazi. Prikazane so s spremljevalne vrste, ki se pojavljajo enkrat, in z razredom frekvence $\geq$ II.

\begin{tabular}{|c|c|c|c|c|c|c|c|}
\hline & cover range & pr. & fr. & & cover range & pr. & fr. \\
\hline sp. of Solano-Polygonetalia & & & & sp. of Stellarietea mediae & & & \\
\hline Setaria viridis & $+-2 \mathrm{a}$ & 35 & 88 & Digitaria sanguinalis & +-3 & 29 & 73 \\
\hline Chenopodium album & $r-2 a$ & 26 & 65 & Erigeron sumatrensis & $r-1$ & 26 & 65 \\
\hline Solanum nigrum & $\mathrm{r}-1$ & 19 & 48 & Erigeron canadensis & $+-2 \mathrm{a}$ & 21 & 53 \\
\hline Amaranthus retroflexus & $r-2 b$ & 19 & 48 & Bidens bipinnata & $r-2 b$ & 11 & 28 \\
\hline Amaranthus hybridus agg. & $r-2 a$ & 15 & 38 & Eleusine indica & $+-2 \mathrm{a}$ & 6 & 15 \\
\hline Portulaca oleracea & $\mathrm{r}-2 \mathrm{a}$ & 13 & 33 & Tragus racemosus & +-1 & 2 & 5 \\
\hline Sorghum halepense & + & 4 & 10 & Chamaesyce nutans & $\mathrm{r}-+$ & 2 & 5 \\
\hline Eragrostis cilianensis & + & 3 & 8 & Helminthotheca echioides & $\mathrm{r}-+$ & 2 & 5 \\
\hline Amaranthus graecizans & $\mathrm{r}-+$ & 3 & 8 & Artemisia annua & + & 1 & 3 \\
\hline Setaria pumila & + & 3 & 8 & Abutilon teophrasti & $\mathrm{r}$ & 1 & 3 \\
\hline Setaria verticillata & + & 2 & 5 & & & & \\
\hline Tribulus terrestris & + & 1 & 3 & other & & & \\
\hline Heliotropium europaeum & + & 1 & 3 & Chamaesyce prostrata & +-1 & 25 & 63 \\
\hline Solanum villosum & + & 1 & 3 & Polygonum aviculare agg. & +-3 & 24 & 60 \\
\hline Galinsoga quadriradiata & + & 1 & 3 & Verbena officinalis & +-1 & 14 & 35 \\
\hline Echinochloa crus-galli & $\mathrm{r}$ & 1 & 3 & Clinopodium nepeta s.l. & +-1 & 8 & 20 \\
\hline
\end{tabular}


Table 5: Synoptic table of Mercurialetum annuae from Southern and Central Europe. For abbreviations see Figure 4.

Tabela 5: Sinoptična tabela asociacije Mercurialetum annude iz južne in Srednje Evrope. Okrajšave kot pri Sliki 4.

\begin{tabular}{|c|c|c|c|c|c|c|c|c|c|c|c|c|c|}
\hline geographical area & Fri & Mar & NL & $\mathrm{D}$ & SLO & $\mathrm{CZ}$ & geographical area & Fri & Mar & NL & $\mathrm{D}$ & SLO & $\mathrm{CZ}$ \\
\hline total relevés $\left(\mathrm{n}^{\circ}\right)$ & 10 & 7 & 5 & 401 & 41 & 180 & Vicia sativa agg. & 40 & . & 20 & . & 5 & . \\
\hline \multicolumn{7}{|c|}{ diagnostic species of Mercurialetum annuae } & Geranium dissectum & . & 29 & . & 11 & 20 & 3 \\
\hline Mercurialis annua $(\mathrm{O})$ & 90 & 100 & 100 & 100 & 68 & 17 & Helminthoteca echioides & . & 57 & . & . & $\cdot$ & . \\
\hline Chenopodium album $(\mathrm{O})$ & 40 & 57 & 100 & 89 & 71 & 89 & Crepis setosa & 50 & . & . & . & . & . \\
\hline Amaranthus retroflexus $(\mathrm{O})$ & 50 & 100 & . & . & 78 & 89 & Diplotaxis muralis & 10 & . & . & 11 & 27 & . \\
\hline Senecio vulgaris $(\mathrm{C})$ & 30 & 57 & 100 & 65 & 44 & . & Calendula arvensis & . & 43 & . & . & $\cdot$ & . \\
\hline Solanum nigrum $(\mathrm{O})$ & 90 & 57 & . & 37 & 54 & 48 & Allium vineale & 40 & . & . & . & 2 & . \\
\hline Echinochloa crus-galli $(\mathrm{O})$ & . & 43 & . & 27 & 24 & 50 & Raphanus raphanistrum & . & . & 20 & 11 & 2 & 7 \\
\hline Galinsoga parviflora (C) & . & . & 20 & 23 & 24 & 38 & Avena fatua & . & 29 & . & . & . & 11 \\
\hline Amaranthus hybridus agg. (O) & . & . & . & . & 22 & 46 & Misopates orontium & 10 & 29 & . & 1 & . & . \\
\hline \multirow{2}{*}{ Chenopodium hybridum $(\mathrm{O})$} & \multirow{2}{*}{. } & \multirow{2}{*}{. } & \multirow{2}{*}{. } & \multirow{3}{*}{15} & & \multirow{3}{*}{46} & Myosotis arvensis & . & $\cdot$ & . & 23 & 2 & 7 \\
\hline & & & & & & & Anchusa arvensis & . & . & . & 1 & 29 & . \\
\hline sp. of Veronico-Euphorbion & & & & & & & Avena barbata & 30 & . & . & . & . & . \\
\hline Euphorbia helioscopia & 30 & . & 80 & 70 & 51 & 37 & Senecio vulgaris & 30 & . & . & . & . & . \\
\hline Fumaria officinalis & 40 & . & 80 & 30 & 17 & 4 & sp. of Artemisietea vulgaris & & & & & & \\
\hline Euphorbia peplus & 30 & . & 40 & 22 & $\cdot$ & $\cdot$ & Elymus repens & 50 & . & 80 & 46 & 29 & 47 \\
\hline Thlaspi arvense & \multirow[t]{2}{*}{10} & \multirow[t]{2}{*}{. } & \multirow[t]{2}{*}{. } & \multirow[t]{2}{*}{21} & \multirow[t]{2}{*}{. } & \multirow[t]{2}{*}{24} & Daucus carota & 70 & 57 & . & 8 & . & 6 \\
\hline sp. of Solano-Polygonetalia & & & & & & & Linaria vulgaris & 60 & 43 & . & . & . & 3 \\
\hline Sonchus asper & 40 & 86 & 80 & 75 & 24 & 32 & Picris hieracioides & 60 & 29 & . & . & . & . \\
\hline Sonchus oleraceus & 80 & . & 60 & 62 & 39 & 39 & Artemisia vulgaris & 30 & . & 20 & 3 & . & 31 \\
\hline Fallopia convolvulus & . & . & 100 & 36 & 27 & 25 & Lapsana communis & . & . & 40 & 11 & . & 5 \\
\hline Setaria viridis & 50 & 100 & . & . & 20 & 18 & Rumex pulcher & . & 29 & . & . & . & . \\
\hline Lamium purpureum & . & $\cdot$ & 60 & 45 & 17 & 24 & sp. of Molinio-Arrhenatheretea & & & & & & \\
\hline Setaria pumila & . & . & . & 15 & 41 & 16 & Taraxacum sect. Ruderalia & . & . & 20 & 35 & 46 & 52 \\
\hline Lamium amplexicaule & . & . & 20 & 19 & 10 & 17 & Rumex crispus & 80 & . & . & . & 17 & 8 \\
\hline Erodium cicutarium & . & $\cdot$ & 20 & 13 & 15 & 10 & Pastinaca sativa & 40 & 43 & . & . & . & . \\
\hline Heliotropium europaeum & . & 43 & $\cdot$ & . & . & $\cdot$ & Ranunculus repens & 20 & . & . & 25 & 10 & 6 \\
\hline sp. of Stellarietea mediae & & & & & & & Poa sylvicola & 50 & . & . & . & 2 & . \\
\hline Convolvulus arvensis & 100 & 86 & 60 & 81 & 61 & 47 & Lolium multiflorum & . & 43 & . & . & . & . \\
\hline Veronica persica & 70 & 100 & 20 & 76 & 80 & 39 & Geranium molle & 40 & . & $\cdot$ & . & . & . \\
\hline Cirsium arvense & 80 & 71 & 80 & 59 & 39 & 49 & Potentilla anserina & $\cdot$ & . & 40 & . & . & . \\
\hline Stellaria media & 50 & . & 100 & 68 & 76 & 47 & Lolium perenne & 30 & . & . & 5 & . & . \\
\hline Capsella bursa-pastoris & 50 & . & 80 & 68 & 46 & 52 & others & & & & & & \\
\hline Persicaria maculosa & 10 & $\cdot$ & 80 & 60 & 49 & 11 & Polygonum aviculare agg. & 50 & 43 & . & 50 & 29 & 43 \\
\hline Anagallis arvensis & 50 & 57 & $\cdot$ & 46 & . & 31 & Poa annua & 50 & . & 80 & 17 & 15 & 20 \\
\hline Sinapis arvensis & 10 & . & 40 & 44 & 12 & 21 & Equisetum arvense & 20 & . & 80 & 34 & 10 & 9 \\
\hline Atriplex patula & . & . & 60 & 26 & 10 & 22 & Calystegia sepium & 60 & . & 40 & . & 44 & . \\
\hline Veronica agrestis & . & . & 100 & 1 & . & . & Galium aparine & . & . & 40 & 44 & . & 29 \\
\hline Mentha arvensis & . & . & 80 & 15 & . & 5 & Persicaria amphibia & . & . & 80 & . & . & . \\
\hline Veronica polita & . & . & 20 & 49 & 2 & 24 & Lactuca serriola & 20 & 43 & . & . & . & 17 \\
\hline Anisantha sterilis & 80 & . & . & . & 7 & . & Chenopodium polyspermum & . & 14 & . & 22 & 17 & 21 \\
\hline Diplotaxis erucoides & . & 86 & . & . & . & . & Plantago major & . & . & . & 38 & 10 & 26 \\
\hline Digitaria sanguinalis & 40 & 29 & . & . & 17 & . & Erysimum cheiranthoides & . & . & 60 & 6 & . & 7 \\
\hline Matricaria inodora & . & . & 20 & 9 & . & 51 & Persicaria lapathifolia & . & . & . & 23 & 12 & 27 \\
\hline Viola arvensis & . & $\cdot$ & 40 & 10 & 5 & 24 & Stachys palustris & . & . & 40 & . & 5 & 5 \\
\hline Portulaca oleracea & 20 & 57 & . & 1 & . & . & Cichorium intybus & . & 43 & . & . & . & 3 \\
\hline Papaver rhoeas & . & . & 40 & 15 & 5 & 13 & Medicago lupulina & . & . & . & 9 & 20 & 11 \\
\hline Aristolochia clematitis & 50 & . & . & . & 17 & . & Medicago sativa & 30 & . & . & . & . & . \\
\hline
\end{tabular}

